

\section{THE NATIONAL BUREAU OF STANDARDS}

\section{Functions and Activities}

The functions of the National Bureau of Standards are set forth in the Act of Congress, March 3, 1901, as amended by Congress in Public Law 619, 1950. These include the development and maintenance of the national standards of measurement and the provision of means and methods for making measurements consistent with these standards; the determination of physical constants and properties of materials; the development of methods and instruments for testing materials, devices, and structures; advisory services to government agencies on scientific and technical problems; invention and development of devices to serve special needs of the Government; and the development of standard practices, codes, and specifications. The work includes basic and applied research, development, engineering, instrumentation, testing, evaluation, calibration services, and various consultation and information services. Research projects are also performed for other government agencies when the work relates to and supplements the basic program of the Bureau or when the Bureau's unique competence is required. The scope of activities is suggested by the listing of divisions and sections on the inside of the back cover.

\section{Publications}

The results of the Bureau's work take the form of either actual equipment and devices or published papers. These papers appear either in the Bureau's own series of publications or in the journals of professional and scientific societies. The Bureau itself publishes three periodicals available from the Government Printing Office: The Journal of Research, published in four separate sections, presents complete scientific and technical papers; the Technical News Bulletin presents summary and prcliminary reports on work in progress; and Basic Radio Propagation Predictions provides data for determining the best frequencies to use for radio communications throughout the world. There are also five series of nonperiodical publications: Monographs, Applied Mathematics Series, Handbooks, Miscellaneous Publications, and Technical Notes.

Information on the Bureau's publications can be found in NBS Circular 460, Publications of the National Bureau of Standards (\$1.25) and its Supplement (\$1.50), available from the Superintendent of Documents, Government Printing Office, Washington 25, D.C. 
LNITED STATES DEPARTMENT OF COMMERCE • Frederick H. Mueller, Secrelary NATIONAL BUREAU OF STANDARDS - A. V. Astin, Director

\title{
Precise Measurement of Heat of Combustion With a Bomb Calorimeter
}

\author{
R. S. Jessup
}

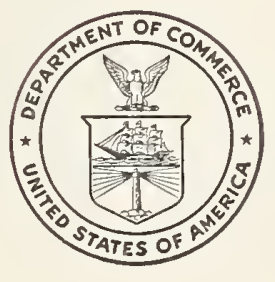

National Bureau of Standards Monograph 7.

Issued February 26, 1960 



\section{Contents}
1.1. Definitions of terms
a. Heat units
b. Heats of combustion

2. General discussion of bomb-calorimetric measure-

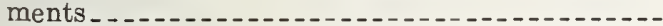

3. Factors affecting accuracy in bomb-calorimetric measurements

3.1. Undesirable side reactions

a. Incomplete combustion....

b. Oxidation of crucible and fittings...-

c. Reaction of acids with bomb material

d. Combustible impurities in oxygen

3.2. Experimental techniques of individual measurements_..............................

a. Weight of sample of combustible.....

b. Weight of calorimeter plus water....

c. Temperature measurements _......

d. Firing energy

e. Materials in bomb

f. Acids formed in combustion

4. Summarized directions for a bomb-calorimetric experiment._._._._._._._._._. 10

4.1. Preliminary adjustment of apparatus_._-_- 10

4.2. Preparation and weighing of sample of benzoic acid

4.3. Preparation of bomb

4.4. Weighing of calorimeter plus water-_._._- 10

4.5. Assembly of calorimeter........... 10

4.6. Adjustment of initial temperature of calorimeter.......................... 11

4.7. Observation of temperature and ignition of sample .

4.8. Analysis of contents of bomb-1

4.2. a. Preparation and weighing of sample of fuel
4. Summarized directions for a bomb-calorimetric experiment-Continued

4.3. a. Preparation of bomb_-_._. 11

4.6. a. Adjustment of initial temperature of

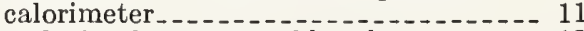

4.8. a. Analysis of contents of bomb_...

5. Calculation of results

5.1. Calibration experiment_...... 12

a. Heat of combustion of benzoic acid

b. Correction terms $c_{1}$ and $c_{2 \ldots} \ldots \ldots$

c. Corrected temperature rise......... 13

d. Example illustrating the calculations $E$ and $E_{8}$

5.2. Calculation of heat of combustion_._._._. 15

a. Energy equivalent of calorimeter as used

b. Corrected temperature rise

c. Corrections $c_{1}, c_{2}, c_{3} \ldots \ldots \ldots$

d. Calculation of $Q_{0}$ (gross) $\ldots \ldots$

e. Calculation of $Q_{p}$ (net)

6. References

7. Appendix

7.1. Apparatus ....... 17

a. Bridge

b. Thermometer

c. Galvanometer.... 18

d. Bomb_....

e. Calorimeter and jacket.

f. Balance for weighing samples....... 20

g. Balance for weighing calorimeter...- 20

h. Oxygen purifier

i. Laboratory table for bridge..... 21

j. Pressure gage........ 21

7.2. Glass sample bulbs _._. 



\title{
Precise Measurement of Heat of Combustion With a Bomb Calorimeter ${ }^{1}$
}

\author{
R. S. Jessup
}

This Monograph gives detailed descriptions of apparatus and methods which are used at the National Bureau of Standards for precise determinations of heats of combustion of liquid hydrocarbon fuels. Numerical examples are given of methods of caleulating results of measurements from observed data. The technique of making and filling glass bulbs to contain samples of volatile liquid fuels is described.

The accuraey of the methods described is about 0.1 percent. This is intermediate between the accuracy of 0.01 or 0.02 percent attained in certain measurements on pure compounds, and the accuracy of several tenths of one percent obtainable with published standard procedures for measurements on fuels.

\section{Introduction}

Standard methods of moderate precision for bomb-calorimetric measurement of heats of combustion of solid and liquid fuels are published by the American Society for Testing Materials [1,2].2 These methods can be used to measure heat of combustion with an accuracy of several tenths of one percent. Apparatus and methods for measurements of heat of combustion with an accuracy of 0.01 or 0.02 percent have been described in the literature [3]. The attainment of such accuracy involves the use of rather laborious and time-consuming procedures. In some applications of fuels the heat of combustion is required with an accuracy of approximately 0.1 percent, which is probably not attainable with the standard methods referred to $[1,2]$, but can be attained with less expenditure of time and effort than is required in the most precise measurements.

This Monograph describes methods and apparatus used at the National Bureau of Standards in connection with several series of investigations of heats of combustion of aircraft fuels $[4,5,6,7]$. The restrictions of the treatment to a particular set of apparatus, and a particular experimental procedure involves some loss of generality, but this is not believerl to be serious. Most precise measurements of heats of combustion of materials containing essentially only the elements carbon, hydrogen, nitrogen, and oxygen have been made in apparatus of similar design, to which the same procedures are applicable. Descriptions of other equally satisfactory apparatus for this purpose will be found in reference [3] and the references there cited. There will also be found in some of these references descriptions of apparatus and procedures suitable for measurements on materials containing considerable amounts of such elements as sulfur and halogens. The apparatus and methorls described in this Monograph are not suitable for measurements on such materials.

Although the apparatus described in this Nonograph is suitable for measurement of heat

1 The preparation of this Monograph was supported in part by the Air Force Ballistic Missiles Division, Air Research and Development Command, U.S. A ir Force.

${ }_{2}$ Figures in brackets indicate the literature references on page 16. of combustion with an accuracy of 0.01 or 0.02 percent, the procedure in measurements on fuels is such that a considerable saving in time was achieved at the cost of a somewhat lower precision. This lower precision resulted from several factors as follows:

(1) As a rule only two experiments were made on each fuel, as compared with six or more experiments where the highest possible accuracy is desired; (2) an approximate method was used to obtain the corrected temperature rise of the calorimeter, i.e., the temperature rise corrected for heat of stirring and heat transfer between calorimeter and surroundings; (3) because some liquid fuels are quite volatile there may be an appreciable loss of lighter components in handling. In spite of these effects the results of measurements even on the most volatile fuels (gasolines) are, in general, precise to about 0.05 percent. The results contain a nearly constant systematic error of approximately 0.03 percent (for hydrocarbon fuels) due to neglect of the Washburn correction [18] which takes account of the difference between heats of combustion in oxygen under a pressure of about $30 \mathrm{~atm}$ and at a pressure of $1 \mathrm{~atm}$.

(If desired, this error can be approximately corrected, in the case of hydrocarbon fuels only, by reducing the observed heat of combustion by 0.03 percent.)

In other respects the methods described are applicable in measurements of the highest precision, and may serve as a useful guide to persons interested in such measurements.

\subsection{Definitions of Terms}

\section{a. Heat Units}

The heat units used in connection with measurements of heats of combustion of fuels are the IT calorie $^{3}$ and the Btu. These units are here defined as follows:

${ }^{3}$ IT calorie is an abbreviation for International Steam Tables calorie. This calorie was adopted by the International Steam Table Conference held in London in July 1929 [12], and is in fairly general use in engineering practice both in this country and abroad. 
1 IT calorie $=4.1868$ absolute joules $1 \mathrm{Btu}=1055.07$ absolute joules.

The definition of the Btu was obtained from that of the IT calorie by means of the relations

1 pound (avoirdupois) $=453.5924$ grams

1 IT calorie per gram=1.8 Btu per pound.

\section{b. Heats of Combustion}

The quantity directly measured in a bombcalorimetric experiment is generally referred to as the "total (or" gross) heat of combustion at constant volume"; this quantity is represented by the symbol $Q_{v}$ (gross) in this Monograph. A precise definition of this term requires a specification of the initial states of the reactants (oxygen and fuel) and of the final states of the products of combustion. For most purposes the following definition is sufficient: The total (or gross) heat of combustion at constant volume of a liquid or solid fuel containing only the elements carbon, hydrogen, oxygen, nitrogen and sulfur is the quantity of heat liberated when unit weight of the fuel is burned in oxygen in an enclosure of constant volume, the products of combustion being gaseous $\mathrm{CO}_{2}, \mathrm{~N}_{2}$, and $\mathrm{SO}_{2}$ and liquid $\mathrm{H}_{2} \mathrm{O}$, with the initial temperature of fuel and oxygen and the final temperature of the products of combustion at $25^{\circ} \mathrm{C}$.

Although the total heat of combustion is the quantity directly measured in a bomb-calorimetric experiment, the quantity required in many practical applications is the "net heat of combustion at constant pressure." This quantity is designated by the symbol $Q_{p}$ (net), and may be defined as follows: The net heat of combustion at constant pressure of a liquid or solid fuel containing only the elements carbon, hydrogen, oxygen, nitrogen, and sulfur is the quantity of heat liberated when unit weight of the fuel is burned in oxygen (or air) at a constant pressure of one atmosphere, the products of combustion being $\mathrm{CO}_{2}, \mathrm{~N}_{2}, \mathrm{SO}_{2}$, and $\mathrm{H}_{2} \mathrm{O}$, all in the gaseous state, with the initial temperature of fuel and oxygen and the final temperature of products of combustion at $25^{\circ} \mathrm{C}$.

For the most fuels to which these definitions apply the elements oxygen, nitrogen, and sulfur will be minor constituents if present at all. The methods described in this Monograph are not suitable for accurate measurements on materials containing more than about 2 percent sulfur.

\section{General Discussion of Bomb-Calorimetric Measurements}

A bomb calorimeter consists essentially of a calorimeter vessel containing a measured amount of water, in which are immersed (1) a thermometer for measuring the temperature of the water, (2) a stirring device for maintaining the water at a uniform, and therefore definitely measurable temperature, and (3) a "bomb" of constant volume in which combustible materials can be burned in oxygen under pressure. In order to control heat transfer between the calorimeter and its environment ("thermal leakage") the calorimeter vessel is enclosed by a "jacket" which is separated from the vessel by an air space about $1 \mathrm{~cm}$ thick, and which for a majority of precise calorimeters, is kept at constant temperature by means of a thermostat.

A schematic diagram of a bomb calorimeter and its jacket is shown in figure 1 . The calorimeter vessel is made in the form shown in order to facilitate stirring of the calorimetric liquid. The effectiveness of a screw propeller stirrer is greatly increased by enclosing it in a tube which extends from near the top to near the bottom of the calorimeter vessel. Putting the tube outside of the main part of the calorimeter vessel, as shown in figure 1(b), reduces the total volume of the calorimeter necessary for a bomb of given size.

In principle, a measurement of the heat of combustion of a given material consists in comparing the corrected temperature rise of the calorimeter in an experiment in which a known quantity of energy is supplied to it, with that produced in another experiment by combustion

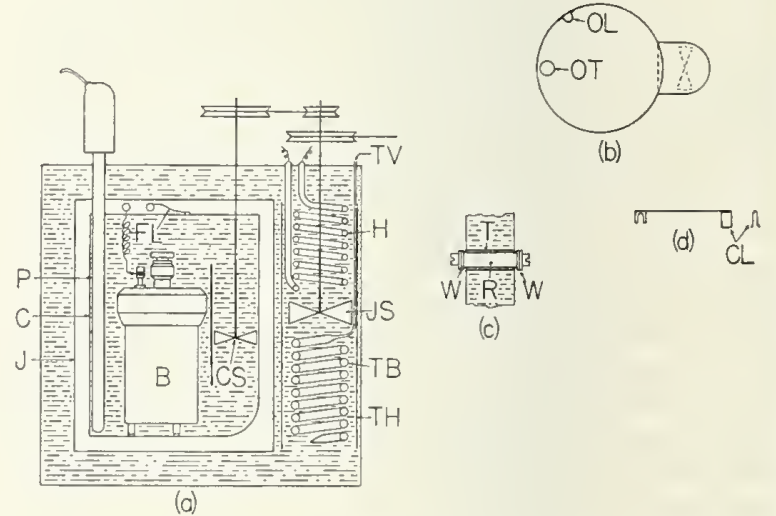

FiguRE 1. Schematic diagram of a bomb calorimeter.

(a) Assembled calorimeter with jacket: B, bomb; C, calorimeter vessel J, jacket wall; P, resistance thermometer; FL, firing leads; CS, calorimeter stirrer; JS, jacket stirrer; TV, tube to thermostat valve; $H$, jacket heater; TB, thermostat bulb; and TH, tubular housing. (b) Top view of calorimeter vessel OL, opening for firing lead; and OT, opening for thermometer. (c) Firing lead through jacket $T$, tube; $R$, rod; and $W$, washers. (d) Firing lead to calorimeter vessel CL, clip.

in the bomb of a weighed sample of the given material. The temperature rise in both experiments should be as nearly as possible over the same temperature range from a standard initial temperature to a standard final temperature. This method of using the corrected temperature rise of the calorimeter to compare an unknown with a known quantity of energy eliminates some of the systematic errors of calorimetric measurements, in particular those associated with uncertainty as to the exact location of the boundary of the 
calorimeter, and those associated with various kinds of lag; by virtue of which different parts of the calorimeter are at different temperatures when the temperature is changing.

Experiments to determine the corrected temperature rise of the calorimeter when a known quantity of energy is supplied to it are generally called calibration experiments, and the mean result of a series of such experiments expressed as energy supplied per unit of corrected temperature rise is called the "energy equivalent" of the calorimeter. The product of the energy equivalent and the corrected temperature rise of the calorimeter in an experiment in which a material of unknown heat of combustion is burned in the bomb gives the energy liberated in the combustion reaction plus any side reactions which may occur.

Calibration experiments are conveniently carried out by burning in the bomb weighed samples of a standard material, such as benzoic acid (NBS Standard Sample 39), the heat of combustion of which is accurately known. If the calorimeter is to be used for measurements of heats of combustion of volatile liquids, such as gasolines, it is desirable to supplement the calibration experiments with benzoic acid by making a series of measurements on NBS Standard Sample 217 of 2,2,4trimethylpentane, which is a volatile liquid with a certified value for its heat of combustion. 'The difference between the measured and certified values of heat of combustion will give an overall check on the accuracy of the measurements, and in particular, on the effectiveness of precautions taken to eliminate effects of volatility.

Since a measurement of heat of combustion involves the comparison of two nearly equal changes in temperature covering the range from a standard initial temperature to a standard final temperature, the temperature scale used in the measurement is not important. Thus temperatures can be expressed in terms of the resistance in ohms of a given platinum resistance thermometer with the energy equivalent of the calorimeter in calories per ohm, or in Celsius degrees with the energy equivalent in calories per degree.

\section{Factors Affecting Accuracy in Bomb-Calorimetric Measurements}

The overall error in the result of a bombcalorimetric measurement is the algebraic sum of individual errors contributed by various factors. Some of the individual errors may be positive and others negative, so that to some extent they may be expected to cancel. However, the extent to which cancellation may take place is uncertain, and may vary from one experiment to another, so that the percentage contribution of each individual error should be kept well below the permissible overall error of the determination. In the present Monograph the permissible difference between duplicate determinations is taken as 0.05 percent. In order to attain this precision, the error contributed by each of the various independent factors should be kept below 0.01 percent so far as possible.

The factors which may affect the accuracy of a bomb-calorimetric determination may be divided roughly into two classes, (1) side reactions which may take place in the bomb, the effect of which cannot be readily evaluated, and (2) the experimental techniques used in making the various individual measurements. These two classes of factors are discussed in sections 3.1 and 3.2 , respectively.

\subsection{Undesirable Side Reactions}

These include (a) formation of carbon monoxide, carbon, or other products of incomplete combustion of the samples; (b) oxidation of the crucible in which combustion of the fuel sample takes place, or oxidation of other parts of bomb or fittings; (c) reaction of acids formed in combustion with the material of the bomb; (d) oxidation of com- bustible impurities such as hydrogen or hydrocarbon gases in the oxygen used.

\section{a. Incomplete Combustion}

In tests of volatile liquid fuels which must be enclosed in glass bulbs to prevent loss by evaporation, incomplete combustion sometimes occurs as a result of breakage of the bulb before ignition of the sample. In such cases large amounts of carbon will usually be formed, and the results of such tests should be discarded. Normally a bulb containing a volatile liquid sample does not break before ignition of the sample, and in such cases there is generally little or no evidence of incomplete combustion. It occasionally happens that globules of glass remaining in the crucible after combustion are gray or black in color. This may be due to metal oxides formed in combustion of the fuse wire, or to inclusion of a small amount of carbon in the glass while it is molten. Determinations of carbon dioxide in the products of combustion of pure liquid hydrocarbons which were enclosed in soft glass bulbs indicate that the amount of carbon in such glass globules does not exceed 0.01 percent of the carbon in the sample.

Incomplete combustion of solid materials, such as benzoic acid, is very likely to occur if these materials are placed in the crucible in powdered form. For this reason, such materials should be compressed into pellets. For samples of nonvolatile liquid fuels which are not enclosed in glass bulbs, and for pelleted samples of benzoic acid, incomplete combustion almost never occurs in apparatus such as that described in the appendix. 


\section{b. Oxidation of Crucible and Fittings}

Oxidation of the crucible and its support and other fittings exposed to the direct action of the flame can be avoided by using platinum crucibles and fittings (see appendix).

\section{c. Reaction of Acids with Bomb Material}

Reaction of acids formed in combustion with the material of the bomb can be made negligible by the use of suitable corrosion-resistant material for the bomb. The bomb described in the appendix has been found satisfactory in this respect. Bombs provided with gold or platinum linings have also been found to be satisfactory.

\section{d. Combustible Impurities in Oxygen}

Combustible impurities in the oxygen used may introduce very serious errors into the results of bomb-calorimetric measurements. Oxygen prepared by electrolysis of water may contain enough hydrogen to cause errors of 1 percent or more. The possibility of error from this source is eliminated by passing the oxygen over copper oxide at $500^{\circ} \mathrm{C}$ before admitting it to the bomb (see appendix).

\subsection{Experimental Techniques of Individual Measurements}

The most important of the individual measurements which go to make up a bomb calorimetric determination inchude measurements of (a) the weight of the sample of combustible; (b) the weight of the calorimeter plus water; and (c) the temperature rise of the calorimeter, including correction for thermal leakage and heat of stirring. A given percentage error in any one of these measurements introduces an equal percentage error into the final result of the experiment. Other individual measurements which determine relatively small correction terms and which can be easily made with the necessary precision include measurements of (d) the quantity of energy used to fire the charge of combustible; (e) the amounts of oxygen and other materials in the bomb; and (f) the amounts of nitric and sulfuric acid formed in the combustion reaction.

\section{a. Weight of Sample of Combustible}

The sample of combustible $(0.8$ to $1.5 \mathrm{~g})$ is weighed with a precision of about $0.02 \mathrm{mg}$ using an undamped semimicro balance with a keyboard arrangement for adding and removing the smaller weights (see appendix). Calibrated high grade (class M) weights are used, and the corrections to the weights given on the calibration certificate are applied in determining the weight of the sample. If weighings are made in the ordinary manner with weights and object weighed on opposite pans of the balance, the balance should be tested to determine whether the balance arms are of equal length. If the arms are found to differ in length by a sionificant amount a correction for this difference will be necessary.

The necessity for such a correction can be eliminated by using the method of weighing by substitution. This consists in placing on the lefthand pan a fixed weight or "tare," somewhat greater in weight than the heaviest object to be weighed, then placing the object to be weighed on the right-hand pan together with sufficient weights, including rider adjustment, to balance against the tare. The object being weighed is then removed and weights and rider again adjusted to obtain balance. The difference in the weights required in the two cases is the desired weight of the object. In addition to eliminating the effect of any difference in length of the balance arms, this method also eliminates any change in sensitivity with load, since the load is constant. The following description of the procedure is for this method of weighing by substitution.

Each half of the balance beam from the center knife edge to the outer knife edge is graduated in 100 equal divisions, so that if a $1 \mathrm{mg}$ rider were used a change in position of the rider by one division on the scale would correspond to a change in weight of $0.01 \mathrm{mg}$. However, it may be inconvenient to use such a small rider because of the likelihood of its being deformed or lost. Instead, a $10 \mathrm{mg}$ rider may be used so that one division on the scale corresponds to $0.1 \mathrm{mg}$ in weight, and hundredths of a milligram obtained from the deflection of the pointer, as described below.

Weighings are normally made with the weights to the nearest milligram on the right-hand pan of the balance, and the $10 \mathrm{mg}$ rider so placed (between 0 and +10 divisions on the beam scale) that the tare weight on the left-hand pan is balanced to the nearest $0.1 \mathrm{mg}$. 'The equilibrium position of the pointer with reference to the zero of the index scale is then determined by observing the turning points of the pointer in an odd number of swings ( 3 or 5$)$.

The procedure in using the balance will be illustrated by an example of the weighing of $x$ sample of benzoic acid. All weighings are made after time has been allowed for temperature equilibrium to be established, and for air currents set up when the balance case was open to die out. To determine when equilibrium has been established, the apparent weight is observed from time to time until no change has been observed during a period of about 10 min.

The following observations were made in determining the weight of a pellet of benzoic acid, the first set of observations were made with the empty crucible, and the second with the crucible containing the pellet: 
Pointer deffection $\times 2,-0.55 \times 0.07 \times 10-3$

Total weights

12. 49316

Crucible plus pellet:

Weights

10. 971

Rider

0.0003

Pointer deflection $\times 2,0.45 \times 0.07 \times 10^{3}$

00003

Total weights

10. 97133

Observed weight of pellet.

1. 52183

Weight correction :

.00005

Corrected weight of pellet

1. 52188

Turning points of swings:

$\begin{array}{ll}-2.6 & 2.0\end{array}$

$-2.5$

$-2.552 .0$

$2 \times$ pointer deflection $=-0.55$

Turning points of swings:

$-2.3 \quad 2.6$

$-2.0$

$-2.152 .6$

$2 \times$ pointer deflection $=+0.45$

- The weight correction is the algehraic sum of the certificate corrections to the individual weights used with the empty crucihle minus the algehraic sum of the corrections to the weights used with crucihle plus pellct.

In the above example the weight corresponding to the pointer deflection from the zero of the index scale was obtained by multiplying twice the average deflection (in divisions on the scale) by one-half of the sensitivity reciprocal of the balance, in this case $0.07=1 / 2 \times 0.14 \mathrm{mg}$ per scale division. (The same result would be obtained, of course, if the average deflection were multiplied by the sensitivity reciprocal, e.g., for the first weighing of the above example the average pointer deflection $=-0.28$ and $-0.28 \times 0.14=-0.04 \mathrm{mg}$ ).

The same procedure is followed, for example, in weighing a sample of a volatile liquid which must be enclosed in a glass bulb to prevent loss by eraporation, except in this case it is not necessary to include the crucible in either weighing. The empty bulb is first weighed against the tare, and then the filled bulb together with glass removed in sealing is weighed against the tare. It should be emphasized that the balancing weight against the tare with only the weights on the righthand pan should be determined in weighing both the empty bulb and the filled bulb. The use of a fixed value for the balancing weight against the tare with only the weights on the right-hand pan would introduce errors if the rest point of the balance changes between the times of weighing the empty and filled bulbs. This is likely to occur if a considerable length of time elapses between the weighings with the bulb empty and filled. The use of a fixed value for the balancing weight with the empty crucible on the right-hand pan is ruled out by the fact that the crucible is subject to changes in weight in use.

\section{b. Weight of Calorimeter Plus Water}

The calorimeter plus water $(3,700 \mathrm{~g}$ total weight $)^{4}$ is weighed on a magnetically damped balance having a capacity of $5 \mathrm{~kg}$ and a sensitivity of $0.5 \mathrm{mg}$ (see appendix). The procedure in this weighing is as follows: The calorimeter is filled with approximately the desired quantity of water, the temperature of which is adjusted to a value such that after assembly of the calorimeter its temperature will be a few tenths of a degree

1 The welght should correspond to an amount of water in the calorimeter such that when the homh is immersed in the water the calorimeter cover can be put in place with its lower side in contact with the water. below the desired initial temperature in the experiment. The choice of the temperature to which the water is to be brought before weighing will depend upon a number of factors, including room temperature, the desired initial temperature in the calorimetric experiment, and the relative heat capacities of calorimeter vessel, water, and bomb. No definite rule can be given for this choice, but the operator will learn by experience how to select the proper temperature under the conditions of his particular laboratory and apparatus.

After adjustment of the temperature the calorimeter is placed on one pan of the balance with the desired weights (see footnote 4) on the other. The amount of water in the calorimeter is adjusted so that the total weight of calorimeter plus water exceeds slightly that of the weights on the opposite balance pan, and the balance case is closed. Because of evaporation of water the weight of calorimeter plus water decreases slowly, and the pointer on the balance gradually moves toward the zero of the index scale. When the pointer reaches the scale zero the balance beam is arrested, the case is opened, and the calorimeter is removed from the balance and placed in position in its jacket preparatory to making a calorimetric experiment.

It is not feasible in weighing the calorimeter to wait for it to come to temperature equilibrium with the air, partly because this would require a very long time and partly because the desired initial temperature in the calorimetric experiment will usually differ from room temperature. Any difference in temperature between calorimeter and the air in the balance case will cause convection currents which will affect the observed weight. The effect of this will partially cancel if the difference between the temperature of calorimeter and the air in the balance case when the calorimeter is weighed is the same in all experiments, including both calibration experiments and measurements of heat of combustion.

The change in weight of the water in the calorimeter due to evaporation after weighing will affect the energy equivalent of the calorimeter, but the effect of this is small and will cancel if the 
procedure in placing the calorimeter in its jacket and completing the assembly of the system is carried out in the same manner and in the same length of time in the calibration experiments as in measurements of heat of combustion.

In addition to the water in the calorimeter vessel, $1 \mathrm{~cm}^{3}$ of water is placed in the previously dried bomb before each experiment to insure that the space in the bomb is saturated with water vapor at the beginning of the experiment as well as at the end, when water formed in combustion will be present in any case. This water is introduced into the bomb by means of a buret or pipet accurate to about $0.01 \mathrm{~cm}^{3}$, immediately before introducing the sample of combustible.

\section{c. Temperature Measurements}

The temperature rise of the calorimeter in a combustion experiment is approximately $3^{\circ} \mathrm{C}$, and must be measured with an accuracy of $0.0003^{\circ}$ if the error in temperature measurement is to contribute not more than 0.01 percent to the result of the calorimetric experiment. Measurements of temperature differences with this precision can be made conveniently with a suitable 25 ohm platinum resistance thermometer, and a Mueller bridge of the type commonly designated as $\mathrm{G}-2$.

In precise measurements of temperature with a resistance thermometer it is customary to eliminate the resistance of the thermometer leads completely by making two resistance readings, with the commutator set first in the $N$ position and then in the $R$ position. In bomb calorimetric measurements where only a small temperature difference is to be measured and where time for making the temperature measurements is limited, it is permissible to omit changing the commutator setting. However, if this procedure is followed it is very important that all measurements in all experiments be made with the same commutator setting, since the change in observed resistance per degree change in temperature may be different for the $N$ and $R$ positions of the commutator. The possibility of error due to change in the commutator setting may be eliminated by permanently disconnecting one of the current leads ( $c$ or $t$ ) of the thermometer from the bridge. The thermometer is then effectively a 3-lead thermometer and the bridge can be operated with only one setting of the commutator.

It is also important that the thermometer be immersed to the same depth in all experiments, so that its contribution to the energy equivalent of the calorimeter will be the same in all experiments, and so that the temperature distribution along the leads will be the same.

The observations of the temperature of the calorimeter in a bomb-calorimetric experiment must be made in such a manner as to provide data from which can be derived a value for the temperature rise corrected for thermal leakage and heat of stirring. The following procedure is for a calorimeter with the jacket maintained constant at a standard temperature. ${ }^{5}$

Temperatures of the calorimeter are measured at definite times during three periods: (1) An initial period of 6 to $10 \mathrm{~min}$ during which the temperature change results solely from thermal leakage and heat of stirring; (2) a middle period of about $12 \mathrm{~min}$, at the beginning of which the charge in the bomb is fired, and during which the temperature change is due partly to thermal leakage and heat of stirring but mostly to the heat liberated by the combustion reaction in the bomb-this period continues until the rate of change of temperature has become constant; and (3) a final period of $10 \mathrm{~min}$ or more, during which the temperature change is again due solely to thermal leakage and heat of stirring. It is desirable to choose the initial temperature of the calorimeter so that its final temperature will be slightly below that of the jacket. This reduces the total thermal leakage during the middle period and eliminates heat transfer by evaporation of water from the calorimeter and condensation on the jacket wall which would occur if the temperature of the calorimeter exceeded that of the jacket.

During the initial and final periods the resistance of the thermometer should be measured with the highest possible accuracy, since the overall accuracy of the determination depends directly upon the accuracy of these temperature measurements. During the middle period, because of the very rapid rate of temperature rise, it is not possible to make readings as accurately as during the initial and final periods, but this is not important because the readings of the middle period are used only for calculating the relatively small correction for thermal leakage and heat of stirring.

With a $\mathrm{G}-2$ bridge and a thermometer having a resistance of approximately $25.5 \mathrm{ohms}$ at $0^{\circ} \mathrm{C}$, one step in the last dial of the bridge $(0.0001$ $\mathrm{ohm}$ ) corresponds to approximately $0.001^{\circ} \mathrm{C}$ in temperature. Readings of the bridge are made to the nearest $0.00001 \mathrm{ohm}\left(0.0001^{\circ}\right)$ by interpolating between two adjacent settings of the last dial of the bridge. 'This interpolation is accomplished by observing the deflection of the galvanometer as follows: The galvanometer is located about 2 meters from a ground glass scale graduated in millimeters, which is so placed as to be conveniently observable by the operator of the bridge. In front of the galvanometer mirror is placed a lens of such focal length that the reflected image of the vertical filament of a light source is focused on the ground-glass scale. ${ }^{6}$ The bridge current is adjusted so that with constant thermometer resistance a change of $0.001 \mathrm{ohm}$ in the bridge

\footnotetext{
${ }_{5}$ The NBS calorimeter is located in an air-conditioned laboratory, the temperature of which is maintained at about $25^{\circ} \mathrm{C}$. The jacket temperature is always maintained constant at $28^{\circ} \mathrm{C}$ and the calorimetric experiments ture is always maintained constant at $28^{\circ} \mathrm{C}$ and the calorimetric experiments
cover the range from $25^{\circ}$ to $28^{\circ} \mathrm{C}$. If room temperature is not controlled the cover the range from $25^{\circ}$ to $28^{\circ}$ C. If room temperature is not controlled the will be above room temperature at all seasons. A jacket temperature below that of the room is undesirable as it may resuit in the condensation of water on the calorimeter, the initial temperature of which is $3^{\circ} \mathrm{C}$ below that of the jacket.

o Lamp and scale devices for indicating galvanometer deflection are available commereially, and are as satisfactory as the device described here.
} 
setting causes a galvanometer deflection corresponding to a $2.5 \mathrm{~cm}$ displacement ${ }^{7}$ of the filament image on the scale. This is equivalent to a displacement of $2.5 \mathrm{~mm}$ per step in the last dial of the bridge, i.e., to $2.5 \mathrm{~mm}$ per millidegree. 'This sensitivity is doubled by reversing the current through the bridge and observing the resulting change in position of the filament image. The sensitivity is such that when the bridge current is reversed a displacement of the filament image by $0.5 \mathrm{~mm}$ corresponds to $0.00001 \mathrm{ohm}\left(0.0001^{\circ}\right)$. Reading the bridge by observing the deflection of the galranometer when the bridge current is rerersed not only increases the sensitivity but also reduces the effect of changes in the galvanometer zero. The reversal of the bridge current is conveniently made by means of a double-pole double-throw toggle switch connected in the battery circuit.

The above discussion of the deflection of the galvanometer when the bridge current is reversed tacitly assumes that the resistance of the thermometer remains constant during this operation. In an actual calorimetric experiment the resistance of the thermometer is usually changing with time; in the initial period for example the resistance of the thermometer is increasing at a rate of approximately $0.0007 \mathrm{ohm} / \mathrm{min}$. $\left(0.007^{\circ}\right.$ $\mathrm{C} / \mathrm{min}$ ). During this period readings are made once per minute in such a manner as to give the resistance of the thermometer to the nearest $0.00001 \mathrm{ohm}$ at interrals of exactly $1 \mathrm{~min}$. These readings are made as follows: The switch for reversing the bridge current is thrown in the direction such that as the temperature of the calorimeter rises the image of the filament of the galvanometer light source moves toward the observer's right. The bridge dials are adjusted occasionally so as to keep the fllament image slightly to the left of its zero position. The time is obserred by means of a stop watch, or preferably by means of a combination of stop watch and audible second signals from a standard clock. The position of the filament image is observed to the nearest $0.5 \mathrm{~mm}$ at a time $4 \mathrm{sec}$ before the even minute and the reversing switch is thrown immediately. The position of the image is observed again at 4 sec after the even minute ${ }^{8}$ and the switch is then thrown back to its original position in preparation for later readings. If the second position of the filament image is exactly the same as the first, then the galvanometer deflection on the even minute was zero, and the reading of the bridge is correct with zero in the next place after the reading of the

7 With the particular combination of bridge, thermometer, galvanometer, and galvanometer to scale distance used, the bridge current of $6.8 \mathrm{ma}$ (thermometer current of $3.4 \mathrm{ma}$ ) gives the sensitivity indicated. Much higher bridge current is undesirable because of the increased heating of the thermometer and the consequent increased variation of thermometer resistance with
slight variations in bridge current. A 6-volt low-discharge storage battery slight variations in bridge current. A 6
is a convenient source of bridge current.

${ }_{8}$ The time which should elapse between the two observations of the galvanometer deflection depends upon the free period of the galvanometer. The reading time of an underdamped galvanometer with a relative damping constant of 0.8 or 0.9 is roughly equal to the free period [19]. last dial. If the second position of the filament image is $x \mathrm{~mm}$ to the left (or right) of the original reading, then the reading of the bridge should be increased (or decreased) by $0.00002 x$ ohm to obtain the reading colresponding to the even minute. For example, if the reading of the bridge is $27.3956 \mathrm{ohms}$ and the upper and lower arrows in figure 2(a) represent the initial and final positions, respectively, of the filament image, then the corrected reading of the bridge at the even minute would be 27.39563 ohms. On the other hand the situation represented in figure 2(b) for the same setting of the bridge dials would correspond to a corrected bridge reading of $27.39556 \mathrm{ohms}$.

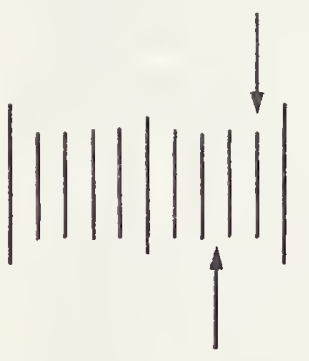

(o)

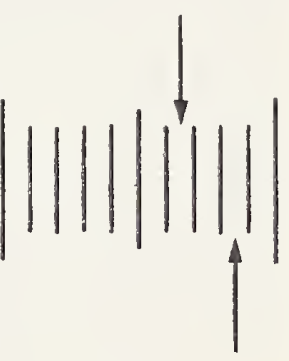

(b)
Figure 2. Diagram to illustrate use of galvanometer deflections in reading bridge.

With a little practice an observer can learn to anticipate each bridge reading fairly closely after the first one or two of a series, and to preset the bridge dials for each subsequent reading so that the change in galvanometer deflection upon reversal of the current will not exceed $2.5 \mathrm{~mm}$ $\left(0.00005 \mathrm{ohm}\right.$ or $\left.0.0005^{\circ} \mathrm{C}\right)$.

As a result of the preliminary adjustment of the temperature of the water in the calorimeter before weighing it as described in section $3.2 . \mathrm{b}$., it is to be expected that after assembling the calorimeter the temperature will be a few tenths of a degree below the desired initial temperature. The temperature should then be raised to about $0.05^{\circ}$ $(0.005 \mathrm{ohm})$ below the temperature at which the initial period is to start, and allowed to drift up to this temperature as a result of thermal leakage and heat of stirring. The observations of the initial period are then made as described above.

The heating of the calorimeter to $0.05^{\circ}$ below the starting temperature is most conveniently done by means of an electric heater which is a permanent part of the calorimeter system. Another method which is frequently used is to place a heated brass rod $(1 / 2 \mathrm{in}$. $\times 2$ in.) on top of the calorimeter cover. The rod is inserted through a hole in the jacket cover which is normally closed by a brass plug. Care is taken to avoid having the heated rod come in contact with the jacket and thereby upset the control of its temperature. 
The above instructions may be illustrated by an example. The calorimeter temperature before weighing was adjusted to a value such that after assembly of the calorimeter the thermometer reading was $20.5+7.420$ ohms. $^{9}$ By means of the heated rod referred to above, the temperature was raised until the reading of the bridge was $20.5+$ 7.465 ohms. The rod was then removed, the plug' replaced in the lole in the jacket, and the calorimeter temperature allowed to drift upward until the bridge reading was $20.5+7.4701$ ohms when the readings of the initial period were begun. These are made once per minute as indicated previously.

Immediately after the observations of the initial period are completed the charge of combustible in the bomb is ignited by means of an electric fuse, the sensitivity of the galvanometer is reduced by reducing the bridge current to about one-third of its original value, and the observations of the middle period are begun. These must be made so rapidly that there is no time for reversing the bridge current for each observation. The observations are made by setting the bridge dials successively at certain predetermined readings and observing the times at which the galvanometer deflection becomes zero. After about 3 min the rate of temperature rise has decreased to such an extent that the bridge current can be increased to its original value and the precise readings of resistance each minute are resumed. These precise readings are continued until the rate of temperature change has been constant for at least $10 \mathrm{~min}$. 'The obser'vations made aftel' the rate of temperature change has become constant constitute those of the final period.

TABLE 1. Observations of time and thermometer resistance during a bomb-calorimetric experiment

\begin{tabular}{|c|c|c|c|c|c|}
\hline $\begin{array}{l}\text { Time } \\
\text { minutes }\end{array}$ & $\begin{array}{l}\text { Resistance } \\
\text { minus } \\
27.5 \text { ohms }\end{array}$ & $\begin{array}{c}\text { Difference } \\
\times 10^{5}\end{array}$ & $\begin{array}{c}\text { Time } \\
\text { minutes }\end{array}$ & $\begin{array}{l}\text { Resistance } \\
\text { minus } \\
27.5 \text { ohms }\end{array}$ & $\begin{array}{l}\text { Difference } \\
\times 10^{5}\end{array}$ \\
\hline $\begin{array}{l}0 \\
1 \\
2 \\
3 \\
4 \\
5 \\
6 \\
6.528 \\
6.621 \\
6.682 \\
6.754 \\
6.820 \\
6.894 \\
6.970 \\
7.066 \\
7.170 \\
7.320 \\
7.399 \\
7.496\end{array}$ & $\begin{array}{c}0.47015 \\
.47078 \\
.47142 \\
.47206 \\
.47334 \\
.47397 \\
.4900 \\
.5100 \\
.5300 \\
.5500 \\
.5700 \\
.5900 \\
.6100 \\
.6300 \\
.6500 \\
.6700 \\
.6800 \\
.6900\end{array}$ & $\begin{array}{r}63 \\
64 \\
64 \\
128 \\
63\end{array}$ & $\begin{array}{l}7.614 \\
7.747 \\
7.922 \\
9 \\
10 \\
11 \\
12 \\
13 \\
14 \\
15 \\
16 \\
17 \\
18 \\
19 \\
20 \\
21 \\
22 \\
23 \\
24 \\
25 \\
26 \\
27 \\
28\end{array}$ & $\begin{array}{r}0.7000 \\
.7100 \\
.7200 \\
.75050 \\
.75920 \\
.76270 \\
.76410 \\
.76470 \\
.76499 \\
.76515 \\
.76525 \\
.76534 \\
.76540 \\
.76546 \\
.76553 \\
.76560 \\
.76566 \\
-. . \\
.76586 \\
.76592 \\
.76599 \\
.76606\end{array}$ & $\begin{array}{r}870 \\
350 \\
140 \\
60 \\
29 \\
16 \\
10 \\
9 \\
6 \\
6 \\
7 \\
7 \\
6 \\
20 \\
7(6) \\
\\
6 \\
6 \\
7 \\
7\end{array}$ \\
\hline
\end{tabular}

8 The bridge has a 20.5 -ohm coil in addition to the 10-, 20-, 30-ohm coils, ete., in the 10x10-ohm decade (see appendix).
Bridge readings made during the course of a typical bomb calorimetric experiment are given in table 1 , and are shown graphically in figure 3 . The values given for resistance represent the observed resistance of the thermometer minus 27.5 ohms. The initial period extends from $t=0$ to $t=6 \mathrm{~min}$, the middle period from $t=6$ to $t=18$ $\mathrm{min}$, and the final period from $t=18$ to $t=28 \mathrm{~min}$. The constancy of the differences between consecutive readings in the initial and final periods is an indication that the system has attained a steady state. This is a necessary condition for the applicability of the method given in section 5 for calculating the corrected temperature rise.

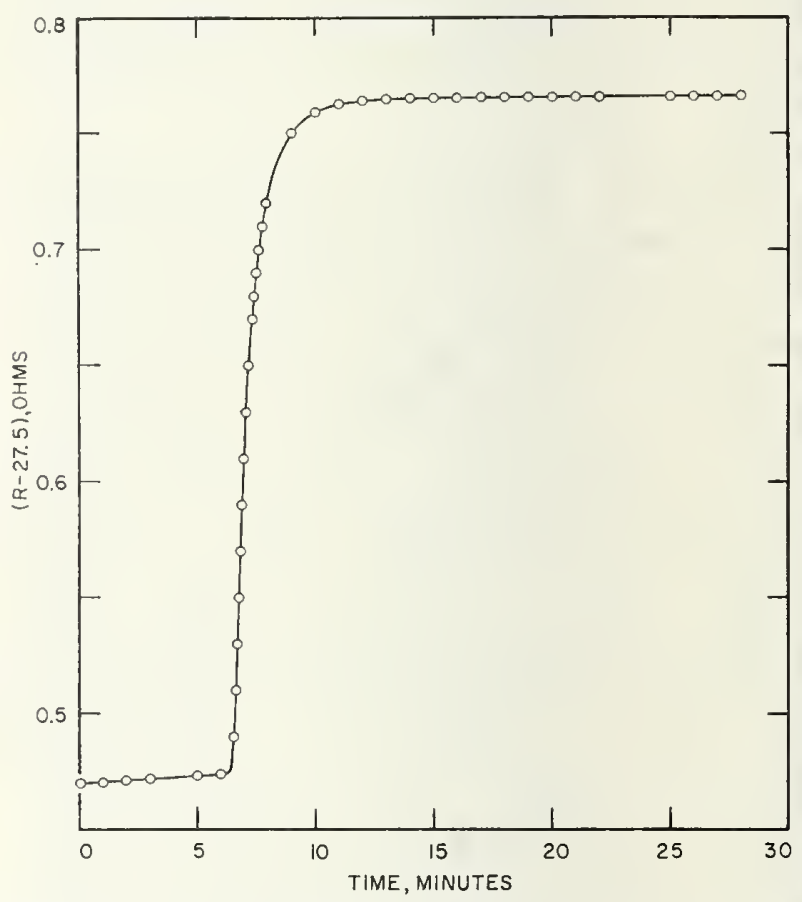

FIGURE 3. Bridge readings versus time in a bomb calorimetric experiment.

In the particular experiment illustrated in table 1 and figure 3 the time readings during that part of the middle period from $t=6$ to $t=9 \mathrm{~min}$ were made by neans of an electric timer which prints the time at which the operator presses a button when he observes the galvanometer defleetion to pass through zero. This instrument is a convenience and would be an important part of the equipment in measurements of extremely high precision. It is not a necessary part of the apparatus for measurements of the precision aimed at in the procedures described in this Monograph. For such precision it is also not necessary to obtain as many readings in the middle period. It is sufficient to make three or four measurements in the range from 55 to 70 percent of the total temperature rise, and to read the time to the nearest second (or the nearest $0.01 \mathrm{~min}$ ) by means of a stopwateh. 
The corrected temperature rise for the experiment illustrated in table 1 and figure 3 is calculated in section 5 .

\section{d. Firing Energy}

The quantity of energy used to fire the charge is the sum of the electric energy used to ignite the fuse plus the heat of combustion of the amount of fuse wire burned. The current for igniting the fuse is obtained from a small transformer with a secondary voltage of about 10 . The total firing energy can be determined in a series of blank experiments in which only the fuse wire is burned. In such experiments the temperature rise of the calorimeter is very small, and the initial temperature of the calorimeter should be very near that of the jacket. The rate of change of temperature in the initial and final periods will then be small, and the correction for thermal leakage will also be small.

When a 2-cm length of fuse wire is used in the manner described in the appendix it has been found that if the wire is burned completely ${ }^{10}$ the firing energy is constant and equal to $5.2 \mathrm{cal}$. No appreciable error will result from using this value in any case where the same type of fuse is used, i.e., a 2-cm length of Parr fuse wire wound into a helix and attached to platinum leads. The firing energy in this case is only 0.05 percent of the energy normally produced in a combustion experiment, and any error in the value $5.2 \mathrm{cal}$ will largely cancel if this same value is used for both calibration experiments and measurements of heats of combustion, and if the temperature rise is about the same in the two kinds of experiment.

The most serious error likely to be encountered in accounting for the firing energy is that which may result from a short-circuit between the firing electrode and the bomb, either because of failure of the insulation around the electrode or because of moisture getting into the mica insulation inside of the bomb. This mica normally is wet by the acid solution formed in combustion and by the water introduced in washing the interior of the bomb after each experiment. It should be dried thoroughly before each experiment, and the insulation should be tested before attaching the fuse to its platinum leads. An insulation resistance of less than $10^{6} \mathrm{ohms}$ usually indicates either moisture in the insulation or some other fallure of the insulating material which needs to be corrected. A "megger" or volt-ohmeter or other similar instrument may be used for testing the insulation resistance.

10 Combustion of the fuse wire Inay not be complete in all blank experiments to determine the firing energy, although it is practically always comments to determine the firing energy, although it is practically always complete When a sample of fuel is burned in the wire is incomplete should be rejected, or else Which combustion of the fusc wire is incomplete shoul
a correction for the unburned wire should be applied.

\section{e. Materials in Bomb}

A calibration of the calorimeter yields a value for the energy equivalent $E$ of the system as actually used, consisting of calorimeter vessel, stirrer, water, thermometer, bomb, and contents of the bomb at the beginning of the experiment. The calorimeter vessel, stirrer, mass of water, thermometer, and bomb will ordinarily be unchanged from one experiment to another, and the crucible and amount of water placed in the bomb will also be constant. However, the mass of the platinum leads to the fuse, the mass of oxygen, and the mass and kind of combustible material may change from one experiment to another. The heat capacity of the largest amount of platinum wire in the bomb amounts to only 0.001 percent of the total energy equivalent of the system, so that variations in the amount of platinum wire are entirely negligible. The mass of oxygen in the bomb is inversely proportional to absolute temperature and directly proportional to absolute pressure. The temperature is measured to about $0.5^{\circ} \mathrm{C}$ at the time the oxygen is admitted to the bomb, by means of a mercurial thermometer with its bulb near the bomb. The pressure of the oxygen (about $30 \mathrm{~atm}=450 \mathrm{psi}$ ) is messured to the nearest $0.1 \mathrm{~atm}(1.5 \mathrm{psi})$ by means of a calibrated Bourdon gage.

The values of the energy equivalent $E$ obtained in calibration experiments are reduced to the energy equivalent $E_{s}$ of a standard calorimeter system consisting of the calorimeter vessel with cover, the standard mass of water, stirrer, thermometer, and bomb containing $1 \mathrm{ml}$ of water, the crucible, and oxygen under an absolute pressure of 30.0 atm at $28.0^{\circ} \mathrm{C}$ but no combustible material. In a measurement of heat of combustion the energy equivalent $E_{s}$ of the standard calorimeter system is corrected for any excess of the oxygen pressure at $28^{\circ} \mathrm{C}$ over 30 atm and for the heat capacity of the charge of combustible.

The reduction of the observed $E$ to the corresponding value of $E_{s}$, and vice versa, is discussed in section 5 on calculation of results.

\section{f. Acids Formed in Combustion}

Nitric acid is normally formed in the combustion reaction from nitrogen either in the fuel sample itself $01^{\circ}$ present as an impurity in the oxygen. If sulfur is present in the fuel, oxides of sulfur will be formed. These are entirely converted to sulfuric acid if nitric acid is also formed. For this reason, if the fuel is known or suspected to contain a significant amount of sulfur, the air initially present in the bomb should not be flushed out with oxygen at the time oxygen is admitted to the bomb. The sulfur will then be all converted to aqueous sulfuric acid, and therefore to a determinate final state.

The total acid formed in combustion is deter- 
mined by washing the inner surface of the bomb with distilled water and titrating the washings against $0.1 N$ sodium hydroxide, using methyl orange as the indicator.

If both nitric and sulfuric acids are formed the total acid is determined by titration as before, and the sulfur is determined by analyzing the bomb washings after titration, using the procedure described in ASTM Designation D129-58 [14]. The correction for nitric acid is $14.0 \mathrm{kcal} / \mathrm{mole}$ of acid formed. If sulfur is present in the fuel the total acid is corrected for as if it were all nitric acid, and an additional correction of $1,400 \mathrm{cal} / \mathrm{g}$ of sulfur is applied.

\section{Summarized Directions for a Bomb-Calorimetric Experiment}

Summarized directions will now be given for carrying out a bomb-calorimetric experiment with benzoic acid to determine the energy equivalent of the calorimeter. Following these directions there will be a brief discussion of the differences in procedure in an experiment to determine the heat of combustion of a fuel.

\subsection{Preliminary Adjustment of Apparatus}

Observe the temperature of the oxygen purifier and if necessary bring its temperature to $500^{\circ} \mathrm{C}$. (Caution: The temperature and oxygen pressure should never be allowed to exceed values consistent with safety with respect to the possibility of explosive failure of the purifier. The manufacturer's recommendations regarding safety precautions should be adhered to rigidly.) Add water, if necessary, to fill the calorimeter jacket bath, bring its temperature to the desired standard value and put the thermostat into opcration (see sec. 3.2.c.).

\subsection{Preparation and Weighing of Sample of Benzoic Acid}

Weigh out approximately an amount of benzoic acid somewhat in excess of $1.52 \mathrm{~g}^{11}$ and compress it into a pellet. Weigh the pellet on a balance sensitive to about $0.01 \mathrm{~g}$ and adjust the weight, if necessary, to $1.52 \pm 0.01 \mathrm{~g}$ (see footnote 11) by scraping the pellet. Carefully remove any powdered benzoic acid from the pellet by brushing. Weigh the crucible on the semimicro balance, first empty and then after placing the pellet of benzoic acid into it, following the procedure described in section 3.2.a.

\subsection{Preparation of Bomb}

Test insulation resistance. If less than $10^{6}$ ohms, dry mica or replace cone insulator.

Attach fuse wire to platinum leads as described in the appendix. ${ }^{12}$ Measure $1.00 \mathrm{~cm}^{3}$ of distilled water into the bomb by means of a buret or pipet. Attach the crucible to its support in such a manner that the fuse is nearly touching the pellet of benzoic acid and assemble the bomb. Connect the bomb

\footnotetext{
11 The standard mass of benzoic acid should be such as to give a temperature rise of approximately $3^{\circ} \mathrm{C}$. The mass used should be witbin $0.01 \mathrm{~g}$ of the standard mass.

${ }_{12}$ It may be convenient to do tbis while waiting for the attainment of temperature equilibrium in the balance case in conncction with weighing the sample of combustible.
}

to the oxygen cylinder through the purifier and admit oxygen to a pressure of approximately 30 atm. The oxygen should be admitted slowly so as not to cool the copper oxide in the oxygen purifier below the temperature at which it will oxidize combustible impurities in the oxygen. Close the valve in the oxygen line and open the needle valve on the bomb, allowing the oxygen to escape until the pressure is reduced to atmospheric. This procedure removes about 97 percent of the nitrogen in the air initially in the bomb. Refill the bomb to the pressure of approximately $30 \mathrm{~atm}$, read and record the temperature and pressure, and disconnect the bomb from the oxygen tank. Attach the binding post (fig. 6(b)) with the lead wire to the terminal nut on the bomb.

\subsection{Weighing of Calorimeter Plus Water}

Fill the calorimeter with water, adjust temperature of water, and weigh calorimeter plus water as described in section 3.2.b.

\subsection{Assembly of Calorimeter}

Inmediately after the calorimeter is weighed carry it to the jacket and place it in its proper position in the jacket space. Place the bomb in the calorimeter, ${ }^{13}$ put the calorimeter cover in place and press it down until its lower surface is in contact with the water. These operations should be performed rapidly and as nearly as possible in the same manner and in the same length of time in all experiments. Complete the firing circuit by connecting the lead to the bomb electrode to the binding post provided for this purpose (fig. 1.(c)) and connect the other lead (already attached to the other binding post) to the calorimeter vessel by means of the clip shown in figure 1.(d) (see appendix). Close the jacket cover and connect the calorimeter stirrer to its driving shaft. Measure the jacket temperature to $0.001^{\circ} \mathrm{C}(0.0001$ $\mathrm{ohm}$ ) by means of the bridge and resistance thermometer, then remove the thermometer from the jacket bath, dry it, and put it in place in the calorimeter.

13 'The bomb should be lowered into the calorimeter' without touching the water with the fingers. This can be donc by using some sort of hook on which the bomb can be hung and which can be remored after the bomb is in place. the bom made of a piece of brass or nichrome rod about $1 / 16$ in. in diameter and bent into the form shown in fig. 6 (f) has been found satisfactory for this pur. bent into the form shown in fig. 6(f) has been found satisfactory for this pur.
pose with the Parr bomb. The hooked ends of the rod are inserted into holes pose with the Parr bomb. The hooked ends of the rod are inserted into holes
on opposite sides of the screw cap of the bomb, and are easily removed after the bomb is in place. The amount of water which adheres to the book when it is withdrawn from the calorimeter is negligible. 


\subsection{Adjustment of Initial Temperature of Calorimeter}

Adjust the calorimeter temperature to $0.05^{\circ}$ $(0.005 \mathrm{ohm})$ below the starting temperature of the initial period, and allow it to drift up to the starting temperature as described in section 3.2.c. The starting temperature (in calibration experiments) always has the same value, which is so chosen that the final temperature of the calorimeter after combustion will be at, or preferably a few hundredths of a degree below, the temperature of the jacket.

\subsection{Observation of Temperature and Ignition of Sample}

After the temperature of the calorimeter has been allowed to drift up to the starting temperature, make and record the readings of time and temperature of the initial period each minute as described in section 3.2.c. Immediately after the last reading of the initial period fire the charge by closing the circuit through the fuse in the bomb. Observe the ammeter in this circuit when the charge is fired. 'The reading of the ammeter' should rise abruptly to about 5 amp and then drop almost instantly to zero. Failure of the cument to drop immediately indicates a short circuit. and the charge will probably not be ignited, or if it is ignited the result of the experiment will be in error by an unknown amount because of the excess electrical energy. If the ignition takes place normally, make and record the observations of the middle and final periods as described in section 3.2.c. After the end of the final period remove the thermometer from the calorimeter, insert it in the jacket bath and measure the temperature of this bath to $0.001^{\circ}$. If the temperature control equipment has been operating properly the jacket temperature should remain constant to $0.005^{\circ}$ or better during an experiment.

\subsection{Analysis of Contents of Bomb}

Remove the bomb from the calorimeter, drs it, open the needle valve and allow the gas to escape at a rate such as to reducc the pressure to atmospheric in not less than 1 min. Then open the bomb and examine the interior for unburned carbon, which would indicate incomplete combustion. If more than a slight trace of unburned carbon is found the experiment should be rejected. If no appreciable amount of unburned carbon is found wash all interior surfaces of the bomb with distilled water and collect the washings quantitatively in a beakel: The quantity of the washings should preferably not cxceed $150 \mathrm{ml}$. Titrate the washings against a $0.1 N$ solution of $\mathrm{TaOH}$ using methyl orange as the indicator. Calculate the correction for nitric acid as desrilibed in section 5 on calculation of results.
The procedure in a measurement of lieat of combustion follows the same steps outlined in paragraphs 4.1 to 4.8 , with certain differences in individual steps as indicated below.

\section{2.a. Preparation and Weighing of Sample of Fuel}

If the material whose heat of combustion is to be determined is a sufficiently nonvolatile liquid, weigh the empty crucible by the method of substitution as described previously, decrease the weights on the pan with the crucible by the desired weight of sample, ${ }^{14}$ and add the liquid drop-wise from a pipet until the weights plus sample plus crucible balance the tare weight on the opposite pan. Ordinarily this procedure will add more than the desired weight of sample, and the excess may be removed, for example, by dipping the end of a fine glass capillary into the liquid. Great care should be taken during the introduction of the sample into the crucible and making the final adjustment of the weight to aroid getting any of the sample on the outside of the crucible. The final weighing of crucible plus sample is carried out as described in section 3.2.a.

If the material whose heat of combustion is to be determined is a volatile liquid, the enclosure of the sample in a glass bulb (as described in the appendix) and the determination of its weight (as described in sec. 3.2.a.) will ordinarily have been done prior to the beginning of the calorimetric measurements. It is then only necessary to place the bulb in the crucible before starting the procedurc described in section 4.3. and 4.3.a.

\section{3.a. Preparation of Bomb}

The procedure here is the same as that described in section 4.3 except that if the sample contains sulfur the air initially in the bomb is not flushed out before filling the bomb with oxygen. If the sample does not contain sulfur the air should be flushed out as described in section 4.3 by filling the bomb with oxygen to $30 \mathrm{~atm}$, allowing the gas to escape until the pressure is reduced to atmospheric, and then refilling the bomb to 30 atm. Where the sample is enclosed in a glass bulb, premature breakage of the bulb when the pressure is first raised to $30 \mathrm{~atm}$ can sometimes be detected by the odor of the fuel in the escaping gas when the pressure is released.

\section{6.a. Adjustment of Initial Temperature of Calorimeter}

In the case of a nonvolatile liquid where the weight of sample is such as to give a tcmperature rise equal to that in a calibration experiment the adjustment of the initial calorimeter temperature is carried out in the samc manner as described in section 4.6.

is The reight of sample should be such as to give approximately the sime temperature rise of the ealorimeter as in the calibration experiments. 
In the case of volatile samples it is difficult to make the glass sample-bulbs sufficiently uniform in capacity to give the same temperature rise of the calorimeter in all experiments. Select the initial temperature for each experiment so that the mean of the initial and final temperatures of the calorimeter will be the same as in the calibration experiments.

\section{8.a. Analysis of Contents of Bomb}

If the sample contains no sulfur follow procedure described in section 4.8. If the sample contains sulfur titrate the bomb washings to determine total acid is described in section 4.8. After this titiation analyze the bomb washings for sulfur using the procedure described in ASTM Designation D129-58 [14].

\section{Calculation of Results}

\subsection{Calibration Experiment}

Calculate a value for the energy equivalent $E$ from the data of each calibration experiment, using the following formula:

$$
E=\frac{Q_{c} \times m_{s}+c_{1}+c_{2}}{\Delta t}
$$

where $Q_{c}=$ heat of combustion of benzoic acid in IT calories per gram under the conditions of the experiment.

$m_{s}=$ weight in air, in grams, of sample of benzoic acid burned.

$c_{1}=$ correction for firing energy, in calories. $c_{2}=$ correction for nitric acid, in calories.

$\Delta t=$ corrected temperature rise of calorimeter, in degrees Celsius (or ohms).

The value of $E$ will be in calories per unit of the temperature scale used, e.g., in calories per $\operatorname{deg} \mathrm{C}$, or calories per ohm.

\section{a. Heat of Combustion of Benzoic Acid}

The certified value for the heat of combustion of the present standard sample (39h) of benzoic acid under the standard conditions of the bomb process is $6318.3 \mathrm{IT}$ cal/g weight in air. ${ }^{15}$ The standar'd conditions are:

A. The combustion reaction is referred to $25^{\circ} \mathrm{C}$.

B. The sample is burned in a bomb of constant volume in pure oxygen at an initial pressure of $30 \mathrm{~atm}$ at $25^{\circ} \mathrm{C}$.

C. The number of grams of sample burned is equal to three times the internal volume of the bomb in liters.

D. The number of grams of water placed in the bomb before combustion is equal to three times the internal volume of the bomb in liters.

If the conditions in the experiment differ from the standard conditions $A, B, C$, and D above, the value of $Q_{c}$ for the heat of combustion of benzoic acid under the actual conditions of the experiment will be obtained from the following approximate equation, which was derived in accordance with the procedure recommended by Washburn [18]:

${ }^{15}$ The value for the heat of combustion of benzoic acid is given here in IT calories per gram weight in air against brass weights, the densities of air brass, and benzoic acid being taken as $0.00116,8.4$, and $1.320 \mathrm{~g} / \mathrm{cm}^{3}$, respec ively

$$
\begin{aligned}
Q_{c}=6318.3\left\{1+10^{-6}\right. & {\left[20(P-30)+42\left(\frac{m_{s}}{V}-3\right)\right.} \\
+ & \left.\left.30\left(\frac{m_{w}}{V}-3\right)-45(t-25)\right]\right\}
\end{aligned}
$$

where $P=$ initial absolute pressure of oxygen, in atmospheres, at the temperature $t$,

$m_{s}=$ weight of sample, in grams,

$m_{20}=$ weight in grams, of water placed in bomb before combustion,

$V=$ internal volume of bomb in liters,

$t=$ temperature to which the combustion reaction is referred, in degrees $\mathrm{C}$.

The temperature to which the reaction is referred is here taken as the final temperature of the calorimeter. The value of the energy equivalent $E$ obtained from eq (1) is then that of the initial calorimetric system at the mean temperature of the calorimeter. By the initial calorimetric system is meant the system before combustion of the benzoic acid, i.e., it includes the benzoic acid, water, and oxygen in the bomb before combustion takes place.

For example let the following data be given:

Volume of bomb,

$$
V \quad=0.380 \text { liter }
$$

Weight of benzoic acid, $m_{s} \quad=1.522 \mathrm{~g} ; m_{s} / V=4.01 \mathrm{~g} /$ liter

Weight of water in bomb, $m_{w}=1.00 \mathrm{~g} ; m_{w} / V=2.63 \mathrm{~g} /$ liter

Initial oxygen pressure

$$
=30.8 \mathrm{~atm} \text { gage at } 25.5^{\circ} \mathrm{C}
$$

Final temperature

$$
=31.8 \mathrm{~atm} \text { abs at } 298.7^{\circ} \mathrm{K}
$$

of calorimeter, $t=28.00^{\circ} \mathrm{C}=301.2^{\circ} \mathrm{K}$

Oxygen pressure

$$
\text { at } \begin{aligned}
28.00^{\circ} \mathrm{C}, P & =31.8 \times \frac{301.2}{298.7} \\
& =32.1 \text { atm absolute }
\end{aligned}
$$

Substituting in eq (2) we obtain

$$
\begin{aligned}
Q_{c}= & 6318.3\left\{1+10^{-6}[20(32.1-30)+42(4.01-3)+\right. \\
& 30(2.63-3)-45(28.00-25.00)]\} \\
= & 6317.9 \mathrm{cal} / \mathrm{g} \text { in air. }
\end{aligned}
$$




\section{b. Correction Terms $c_{1}$ and $c_{2}$}

The correction $c_{1}$ for firing energy is preferably determined in a series of blank experiments as described in section 3.2.d. The use of the value $c_{1}=5.2$ cal will not lead to a significant error if the fuse is a 2-cm length of Parr fuse wire wound into a helix and attached to platinum leads. In some cases a greater length (5 cm or more) of fuse wire is attached to electrodes which are located a short distance away from the crucible. In such cases the fuse may not all burn, and the firing energy will be different in different experiments depending upon the length of wire burned. If this procedure is followed, the length of wire burned in each experiment must be determined and the correction $c_{1}$ may be taken as $2.6 \times L$ calories, where $L$ is the length of wire burned, in centimeters.

The correction $c_{2}$ for nitric acid is $14.0 \mathrm{kcal} / \mathrm{mole}$ of nitric acid formed, or $14.0 \mathrm{~N} \mathrm{cal} / \mathrm{cm}^{3}$ of sodium hydroxide solution of normality $N$ required to neutralize the acid. Thus if $2.4 \mathrm{~cm}^{3}$ of $0.0987 \mathrm{~N}$ sodium hydroxide is required to neutralize the nitric acid formed, $c_{2}=2.4 \times 14.0 \times 0.0987=3.3 \mathrm{cal}$.

\section{c. Corrected Temperature Rise}

The calculation of the corrected change in resistance of the thermometer in a bomb calorimetric experiment will be illustrated by carrying out the calculations for the experiment in which the data given in table 1 were obtained. As stated in section 3.2.c. the initial, middle, and final periods in this experiment extend from $t=0$ to $t=6$, $t=6$ to $t=18$, and $t=18$ to $t=28 \mathrm{~min}$, respectively. The observed change in resistance of the thermometer is taken as the difference between the bridge readings at $t=6$ and $t=18 \mathrm{~min}$, namely, $0.76540-0.47397=0.29143 \mathrm{ohm}$. This difference is to be corrected for thermal leakage and heat of stirring. Correct methods for calculating this correction will be found in reference [3] and in a number of the references there cited. For the accuracy aimed at in this manual it is sufficient to use the following procedure which has been found empirically to give correct results within about 0.01 percent of the total resistance cliange. We first calculate the average rates of change of resistance $r_{1}$ and $r_{2}$ in the initial and final periods, respectively. These are seen to be

$r_{1}=\frac{0.47397-0.47015}{6}=\frac{0.00382}{6}=0.000637 \mathrm{ohm} \mathrm{min}$ $r_{2}=\frac{0.76606-0.76540}{10}=\frac{0.00066}{10}=0.000066 \mathrm{ohm} \mathrm{min.}$

Next we calculate from the data of the middle period the time $t_{m}$ at which 63 percent of the observed lesistance change has taken place. The resistance at $t_{m}$ is given by that at the final reading of the initial period (at $t=6$ ), 0.47397 , plus 63 percent of the observed resistance change. In other words

$$
R_{m}=0.47397+0.63 \times 0.29143=0.65757 \mathrm{ohm} .
$$

Referring to table 1 it is seen that this resistance is reached at a time intermediate between 7.17 and $7.32 \mathrm{~min}$, and by interpolation we obtain $t_{m}=7.227 \mathrm{~min}$. The corrected initial and final resistances, $R_{i}$ (corr.) and $R_{f}$ (corr.), are then obtained by applying corrections to the observed resistance at $t=6$ and $t=18 \mathrm{~min}$ as follows: ${ }^{16}$

$$
\begin{aligned}
R_{i}(\text { corr. })= & R_{6}(\mathrm{obs})+r_{1}\left(t_{m}-6\right) \\
= & 0.47397+0.000637 \times 1.227=0.474752 \\
& \text { ohm }, \\
R_{f}(\text { corr. })= & R_{18}(\mathrm{obs})-r_{2}\left(18-t_{m}\right) \\
= & 0.76540-0.000066 \times 10.773=0.764689 \\
& \text { ohm. }
\end{aligned}
$$

The change in resistance corrected for thermal leakage and heat of stiring is then

$$
\begin{aligned}
R_{f}(\text { corr. })-R_{i}(\text { corr. })= & 0.764689 \\
& -0.474752=0.289937 \mathrm{ohm} .
\end{aligned}
$$

Practically the same result would have been obtained if only three readings had been taken during the period of very rapid temperature rise, say at $R=0.6300,0.6600$, and $0.6900 \mathrm{ohm}$.

This result must be corrected for errors in the bridge coils by applying a correction equal to the algebraic sum of the certificate corrections to the coils used in obtaining $R_{18}$ (obs) less the algebraic sum of the corrections to the coils used in obtaining $R_{6}$ (obs.). It is evident that it is not necessary in this calculation of the change in resistance to include corrections to coils which enter into both $R_{6}$ (obs) and $R_{18}$ (obs). In this case such coils include one of 20.5 ohms and one of 7 ohms. The bridge correction in the experiment under consideration was $+0.000029 \mathrm{ohm}$, making the corrected resistance change $\Delta R=0.289966 \mathrm{ohm}$.

Resistance of the thermometer can be converted to temperature with the aid of the formula or tables accompanying the NBS certificate for the thermometer. It is somewhat more convenient, however, to compute a table of factors $\Delta t / \Delta R$ which when multiplied by the corrected change in resistance $\Delta R$ will give the desired change in temperature $\Delta t$. Such a table may be calculated as follows. Finst the temperatures corresponding to certain given resistances, say $R=27.5000,27.6000$, $\ldots-28.5000 \ldots$, are calculated, using the data in the certificate for the thermometer. These temperatures are then substituted into the formula

$$
\frac{\Delta t}{\Delta R}=\frac{1}{R_{0} \alpha\left[1+\frac{\delta}{100}-\frac{2 \delta t}{10000}\right]}
$$

16 Resistances are read to 1 in the fifth decimal. The calculations are carried one place farther to avold errors from rounding off.

i7 Strictly speaking, eq (3) gives the derivative $d t / d R$. This is exactly equal is strictly speaking, eq (3) gives the derivative $d t / d R$. This is exactly equa $0 \Delta t / \Delta R$ for a finite interval $\Delta R$, provided it is calculated for the mean tem perature of the interval. If, as is customary, the $v a l u e$ of $d t / d R$ corresponds
to the mean resistance of a finite interval, then $d t / d R$ is not exactly equal to $\Delta t / \Delta R$, but the error is entirely negligible. 
where $R_{0}, \alpha$, and $\delta$ are constants given in the calibration certificate for the particular thermometer. The values of $R$ and corresponding values of $\Delta t / \Delta R$ calculated from the above formula are tabulated. The raluc of $\Delta t / \Delta R$ to use in calculating the corrected temperature rise in a given experiment is that corresponding to the mean of the corrected initial and final values of $R$ for the experiment. This value of $\Delta t / \Delta R$ is obtained from the table, by interpolation if necessary. For example, in the example given above the value of $\Delta t / \Delta R$ used should be that corresponding to the mean of $27.5+R_{i}$ (corr.) and $27.5+R_{f}$ (corr.) or

\section{$27.5+(0.47475+0.76469) / 2=27.5+0.6197$} $=28.1197$ olims. $^{18}$

It is found that $\Delta t / \Delta R$ increases by about 0.03 percent for an increase in temperature of $1 \mathrm{deg} C$ $(0.1 \mathrm{ohm})$.

It is not necessary, lowever, to convert resistance to temperature. If desired, the resistance change can be taken as the measure of the temperature change, with the energy equivalent $E^{\prime}$ expressed in caloreis per ohm. The corrected resistance change $\Delta R$ (ohms) in a measurement of heat of combustion would then be multiplied by $E$ (calories per ohm) to give the energy (calories) produced in the combustion reaction. One disadvantage of this procedure is that $E$ in calories per ohm changes more for a given change in the mean temperature of the experiment than does $E$ in calories per degree. For a typical bomb calorimeter it has been calculated that the change in the value of $E$ for an increase of one degree $(0.1 \mathrm{ohm})$ in the mean temperature of the experiment is about +0.03 percent if $E$ is expressed in calories per ohm, but is less than 0.01 percent if $E$ is expressed in calories per degree. Hence if temperatures are expressed in ohms, then for a precision of 0.01 percent, the mean temperature of the calorimeter should be kept within about 0.2 degree $(0.02$ $\mathrm{ohm}$ ) of some fixed value, or else the value of $E_{s}$ in each calibration experiment should be corrected to some standard temperature, and the mean value of $E_{s}$ for this standard temperature should be corrected to the actual mean temperature of each measurement of heat of combustion. The greater change with temperature of $E_{s}$ expressed in calories per ohm is due to the fact that the resistance of the thermometer is not a linear, function of temperature, i.e., the fact that $\Delta t / \Delta R$ is not constant.

A convenient check on the behavior of the calorimeter and the correctness of the temperature measurements may be obtained by calculating the "cooling constant," $k$, of the calorimeter. This is defined by

\footnotetext{
18 Strictly speaking, the corrections to all the coils used should be applied
in calculating the mean value of the resistance. In many cases, however, these corrections are too small to be significant in this calculation and may
} be neglected.

$$
k=-\frac{r_{2}-r_{1}}{R_{2}-R_{1}}
$$

where $r_{1}$ and $r_{2}$ are the avelage rates of change of resistance in the initial and final periods, respectively, and $R_{1}$ and $R_{2}$ are the observed resistances at the mid-points of these periods. Thus, using the data of table 1 at the beginning of this section $(5.1 \mathrm{c})$ :

$$
k=\frac{0.000637-0.000066}{0.76573-0.47206}=0.001944 .
$$

This quantity is a constant of the calorimeter and should remain constant to better than one percent in different experiments.

\section{d. Example Illustrating the Calculations of $E$ and $E_{s}$}

The calculation of $E$ may be illustrated by an example, using the following data taken from preceding sections as indicated.

Weight benzoic acid, $m_{s}=1.52188 \mathrm{~g}$ (sec. $3.2 \mathrm{a}$ )

Heat of combustion of benzoic acid, $Q_{\mathrm{c}}=6317.9$ $\mathrm{cal} / \mathrm{g}$ (sec. 5.1.a)

Firing energy ( $2 \mathrm{~cm}$ fuse wire), $c_{1}=5.2 \mathrm{cal}$ (sec. $5.1 . b)$

Correction for nitric acid, $c_{2}=3.3 \mathrm{cal}$ (sec. 5.1.b)

Corrected resistance change, $\Delta R=0.289966$ ohm (sec. 5.1.c)

Corrected temperature change, $\Delta t=2.87462 \mathrm{deg}$ $\mathrm{C}(\Delta t / \Delta R=9.91364)$

Oxygen pressure at $28.0^{\circ}, P=32.1$ atm abs (sec. 5.1.a)

Substituting the above values in eq (1), the value of $E$ is found to be

$$
\begin{aligned}
E & =\frac{1.52188 \times 6317.9+5.2+3.3}{0.289966}=33188.7 \mathrm{cal} / \mathrm{ohm} \\
& =\frac{1.52188 \times 6317.9+5.2+3.3}{2.87462}=3347.78 \mathrm{cal} / \mathrm{deg} .
\end{aligned}
$$

To reduce the above values of $E$ to the corresponding values of $E_{s}$, the energy equivalent of the standard calorimeter system, it is only necessary to subtract the heat capacity of the sample

\begin{tabular}{|c|c|c|}
\hline & $\mathrm{cal} / /^{\circ} \mathrm{C}$ & $\mathrm{cal} / \mathrm{ohm}$ \\
\hline $\begin{array}{l}\text { Observed value of } E \\
\text { Heat capacity of excess oxygen }=(32.1-30.0) \times 0.077 \\
\text { Heat capacity of benzoic acid }=1.522 \times 0.29=\end{array}$ & $\begin{array}{r}3,347.78 \\
0.16 \\
0.44\end{array}$ & $\begin{array}{r}33,188.7 \\
1.6 \\
4.4\end{array}$ \\
\hline Value of $E_{8}$ & $3,347.18$ & $33,182.7$ \\
\hline
\end{tabular}
of benzoic acid, and of the amount of oxygen in excess of that required to fill the bomb to $30 \mathrm{~atm}$ absolute at $28^{\circ} \mathrm{C}$, as follows:

The factor 0.077 used in calculating the correction for excess oxygen is the increase in heat capacity (in calories per degree) per atmosphere 
increase in pressure in the bomb used, which has an internal volume of 0.380 liter. For a bomb of volume $V$ the corresponding factor is 0.077 $V / 0.380$. The factor 0.29 used in correcting for the benzoic acid is the specific heat of benzoic acid in calories per gram degree Celsius. Both factors are greater by a factor of 10 if $E$ is in calories per ohm (for a thermometer having a resistance of $25.5 \mathrm{ohms}$ at $0^{\circ} \mathrm{C}$ ).

\subsection{Calculation of Heat of Combustion}

Calculate a value of total (or gross) heat of combustion at constant volume from the data of each measurement, using the following formula.

$$
Q_{0}(\text { gross })=\frac{E \times \Delta t-c_{1}-c_{2}-c_{3}}{m_{s}},
$$

where:

Q. (gross) = total heat of combustion at constant volume at the final temperature of the experiment, in IT calories per gram.

$E=$ energy equivalent of the calorimeter as used, in IT cal/deg (or IT cal/ohm)

$\Delta t=$ corrected temperature rise of calorimeter in degrees (or ohms)

$m_{s}=$ mass of sample, in grams

$c_{1}=$ firing energy, in calories

$c_{2}=$ correction for energy of formation of nitric acid, in calories

$c_{3}=$ correction for energy of formation of sulfuric acid, in calories.

a. Energy Equivalent of Calorimeter as Used

The calorimeter as used differs from the standard calorimeter because it contains the sample of fuel and because, in general, the absolute pressure of the oxygen at $28^{\circ}$ differs from $30.0 \mathrm{~atm}$.

The energy equivalent $E$ of the calorimeter as used is obtained by adding to the energy equivalent $E_{s}$ of the standard calorimeter the heat capacity of the charge of combustible and the heat capacity of the oxygen in excess of that required to fill the bomb to $30.0 \mathrm{~atm}$ absolute at $28^{\circ} \mathrm{C}$. The calculation is illustrated as follows, taking the sample of combustible as $0.92231 \mathrm{~g}$ of gasoline, and the oxygen pressure at $28.0^{\circ}$ as 29.0 atm absolute:

\begin{tabular}{|c|c|c|}
\hline & $\mathrm{cal} /{ }^{\circ} \mathrm{C}$ & $\mathrm{cal} / \mathrm{ohm}$ \\
\hline $\begin{array}{l}\text { Energy equivalent of standard calorimeter, } E_{E_{-}=}= \\
\text {Heat capacity of excess oxygen, }(29.0-30.0) \times 0.077= \\
\text { Heat capacity of sample, } 0.922 \times 0.51-\ldots\end{array}$ & $\begin{array}{r}3,347.18 \\
-.08 \\
+.47\end{array}$ & $\begin{array}{r}33,182.7 \\
-0.8 \\
+4.7\end{array}$ \\
\hline Energy equivalent of calorimeter as used, $E=$ & $3,347.57$ & $33,186.6$ \\
\hline
\end{tabular}

The factor 0.51 used in calculating the heat capacity of the sample is the specific heat of gasoline ${ }^{19}$ at $26.5^{\circ} \mathrm{C}$ in cal $/ \mathrm{g}^{\circ} \mathrm{C}$. The factor 0.077 used in calculating the heat capacity of the excess oxygen is defined in section 5.1.d. Both factors are greater by a factor of 10 if $E_{s}$ is in calories per ohm (for a thermometer having a resistance of $25.5 \mathrm{ohms}$ at $0^{\circ} \mathrm{C}$ ). The heat capacity of the glass bulb (less than $0.1 \mathrm{~g}$ ) used to enclose the sample of gasoline may be neglected.

\section{b. Corrected Temperature Rise}

Directions for calculating the corrected temperature rise are given in section 5.1.c. The value to be used in the example to be given later may be taken as $0.309617 \mathrm{ohm}$ or $3.06943^{\circ} \mathrm{C}$.

\section{c. Corrections $c_{1}, c_{2}, c_{3}$}

The correction $c_{1}$ for firing energy with a $2-\mathrm{cm}$ length of fuse wire may be taken as $5.2 \mathrm{cal}$. The corrections $c_{2}$ and $c_{3}$ are calculated as follows: The correction $c_{2}$ is calculated as if all the acid found by titration were nitric acid, as described in section 5.1.b. Thus if it required $14.2 \mathrm{ml}$ of $0.0987 \mathrm{~N}$ sodium hydroxide to neutralize the total acid the correction $c_{2}$ would be given by $c_{2}=$ $14.2 \times 14.0 \times 0.0987=19.6 \mathrm{cal}$. If the test for sulfur showed $0.0042 \mathrm{~g}$ sulfur present in the bomb washings the correction for sulfuric acid, as given in section 3.2 .f., would be $c_{3}=1400 \times 0.0042=5.9$ cal.

\section{d. Calculation of $Q_{v}$ (gross)}

Substituting the values of $E$ in calories per degree (or calories per ohm), $\Delta t$ (or $\Delta R$ ), $c_{1}, c_{2}, c_{3}$, and $m_{s}$ given in sections 5.2.a., b., and c. into formula (4) yields:

$$
Q_{0}(\text { gross })=\frac{3347.57 \times 3.06943-5.2-19.6-5.9}{0.92231}
$$

or

$$
Q_{v}(\text { gross })=\frac{33186.6 \times 0.309617-5.2-19.6-5.9}{0.92231} .
$$

Both of these expressions yield the value 11107.4 $\mathrm{cal} / \mathrm{g}$ weight in air for the total (or gross) heat of combustion of the liquid gasoline at constant volume. The corresponding value in terms of engineering units is

$$
Q_{0}(\text { gross })=11107.4 \times 1.8=19993 \mathrm{Btu} / \mathrm{lb} .
$$

The method of calculation is such that the value obtained for $Q_{0}$ (gross) is referred to the final temperature of the calorimeter, in this case $28.1^{\circ} \mathrm{C}$. The observed value may be reduced to the standard temperature of $25^{\circ}$ by means of the following formula:

19 Taken from NBS Miscellaneous Publication M97 [15] for a gasoline having an API gravity of 70 . Values of heat capacity of petroleum products given in this publication for a temperature of $80^{\circ} \mathrm{F}$ range from 0.42 to 0.52 for API gravities ranging from 10 to 80 . 
Where the value of the factor 1 is given in the following table. ${ }^{20}$

\begin{tabular}{|c|c|c|c|}
\hline$O_{\mathrm{v}}$ (gross) & A & $n_{v}$ (gross) & $A$ \\
\hline$B t v / l b$ & $B t u / l b^{\circ} C$ & Btu/lb & Btu $/ l b^{\circ} C$ \\
\hline 18,500 & 0.68 & 19. 600 & 1. 14 \\
\hline 18,600 & .72 & 19,700 & 1. \\
\hline 18,700 & .76 & 19,800 & 1.2 \\
\hline 18,800 & .80 & 19,900 & 1.2 \\
\hline 18,900 & .85 & 20,000 & 1.3 \\
\hline 19,000 & .89 & 20,100 & 1.3 \\
\hline 19,100 & .93 & 20,200 & 1. \\
\hline 19,200 & .97 & 20,300 & 1.4 \\
\hline 19,300 & 1.01 & 20,400 & 1. 4 \\
\hline 19,400 & 1.05 & 20,500 & 1. 51 \\
\hline 19,500 & 1.09 & 20,600 & 1. 55 \\
\hline
\end{tabular}

Substituting the value of $Q_{v}$ (gross, $\left.28.1^{\circ} \mathrm{C}\right)=$ $19993 \mathrm{Btu} / \mathrm{lb}$ and the corresponding value $A=1.30$ from the above table in eq (5) we obtain

$$
\begin{gathered}
\text { Q. (gross, } \left.25^{\circ} \mathrm{C}\right)=19993+1.30 \times 3.1= \\
19997 \mathrm{Btu} / \mathrm{lb} .
\end{gathered}
$$

29 Tbe values of $A$ werc calculated using the specific beats of liquid petroleum fuels, of gaseous oxygen and carbon dioxide, and of liquid water. The masses of oxygen consumed and of carhon dioxide and water formed in combustion of unit mass of fuel were obtained from an empirical relation between $Q_{\text {。 }}$ (gross) and hydrogen content. See reference [5]. In gencral, the change in $Q_{0}$ gross (Btil/b) with temperature for any fuel may be calculated from tbe relation $d 0_{0}$ (gross) $/ d t=K_{0}$ (reactants) $-K_{\text {。 }}$ (products).

Here $K_{\text {. }}$ (reactants) denotes the heat capacity at constant volume of $1 \mathrm{lb}$ of fuel plus the oxygen consumed in its comhustion, and $K_{v}$ (products) denotes the heat capacity of the products of combustion of one pound of fue] including gaseous carbon dloxide and liquid water. In calculating $K_{\text {o }}$ tbe heat capacities of solids and liquids may be taken as heat capacities at constant pressure.
If the hydrogen content of the fuel is known the value of the net heat of combustion, $Q_{p}$ (net, $25^{\circ}$ ), can be calculated from that for $Q_{0}$ (gross) by means of the following formula [4]:

$$
\begin{gathered}
Q_{p}\left(\text { net, } 25^{\circ} \mathrm{C}\right)=Q_{n}\left(\text { gross, } 25^{\circ} \mathrm{C}\right)- \\
91.23(\% \mathrm{H})
\end{gathered}
$$

Thus if $Q_{0}$ (gross, $25^{\circ} \mathrm{C}$ ) $=19997 \mathrm{Btu} / \mathrm{lb}$ and $(\% \mathrm{H})=13.9$, we obtain

$$
\begin{gathered}
Q_{p}\left(\text { net, } 25^{\circ} \mathrm{C}\right)=19997-91.23 \times 13.9= \\
18729 \mathrm{Btu} / \mathrm{lb} .
\end{gathered}
$$

If the hydrogen content of the fuel is not known, an approximate value for $O_{p}$ (net, $25^{\circ} \mathrm{C}$ ) can be calculated from the following empirical relation:

$$
\begin{gathered}
Q_{p}\left(\text { net, } 25^{\circ} \mathrm{C}\right) \\
=4310+0.7195 Q_{v}\left(\text { gross }, 25^{\circ} \mathrm{C}\right) .
\end{gathered}
$$

Substituting the value $Q$, (gross, $25^{\circ} \mathrm{C}$ ) $=19997$ $\mathrm{Btu} / \mathrm{lb}$ in this equation we obtain

$$
\begin{aligned}
Q_{p}\left(\text { net, } 25^{\circ} \mathrm{C}\right) & =4310+0.7195 \times 19997 \\
& =18698 \mathrm{Btu} / \mathrm{lb}(\text { approximate })
\end{aligned}
$$

Equation (6) is preferable to eq (7) for calculating net from gross heat of combustion, since the latter equation is purely empirical. A fairly accurate value of $(\% \mathrm{H})$ is required in eq (6), since an error of 0.1 in this quantity introduces an error of about 0.05 percent in $Q_{p}$ (net).

\section{References}

[1] ASTM Method D 240-57T. ASTM Standards 7, $143,(1958)$.

[2] ASTM Method D 271-58, ASTM Standards, 8, 999 (1958).

[3] F. D. Rossini, Experimental thermochemistry (Interscience Publishers, New York, N.Y., 1956), and the references there cited.

[t] R. S. Jessup and C. S. Cragoe, Nat. Advisory Comm. Aeronaut. Tech. Note No. 996 (1945) (copies not available).

[5] S. Rothberg and R. S. Jessup, Ind. Eng. Chem. 43, 981 (1951).

[6] R. S. Jessup and J. A. Cogliano, ASTM Bull. No. 201 (Oct. 1954).

[7] G. T. Armstrong, R. S. Jessup, and T. W. Mears, Chem. Eng. Data Ser. 3, 20 (1958).

[8] H. C. Dickinson, Bul. BS 11, 189 (1914) S230.

[9] N. S. Osborne, H. F. Stimson, and T. S. Sligh, Bul. BS 20, 119 (1925) S503.

[10] C. H. Meyers, J. Am. Chem. Soc. 45, 2135 (1923).

[11] T. W. Richards and F. Barry, J. Am. Chem. Soc. 37, 993 (1915)

[12] IT. N. Davis, Mech. Eng. 51, 791 (1929).

[13] See for example:

(a) N. Irving Sax, Handbook of dangerous materials (Reinhold Publishing Corp., New York, N.Y., 1951). (b) Manufacturing Chemists Association, General Safety Committee, Guide for safety in the chemical laboratory (D. Van Nostrand and Co., New York, N.Y., 1954).

(c) National Safety Council, Accident prevention manual for industrial operations (Chicago, Ill., 1951).

(d) National Board of Fire Underwriters, National electrical code (Chicago, Ill., 1940).

[14] ASTM Method D 129-58, ASTM Standards 8, 81 (1955).

[15] C. S. Cragoe, Thermal Properties of Petroleum Products, NBS Misc. Publ. M97 (this paper is out of print but may be found in many public libraries).

[16] E. J. Prosen and F. D. Rossini, J. Research NBS 2\%, 289 (1941) RP1420.

[17] J. Coops, D. Mulder, J. W. Dienske, and J. Smittenberg, Rec. trav. chim. 66, 153 (1947).

[18] E. W. Washburn, J. Research NBS 10, 525 (1933) RP546; see also chapters 5 and 6 of referencc [3].

[19] F. K. Harris, Electrical measurements, p. 57 (John Wiley \& Sons, Inc., New York, N.Y., 1951).

[20] E. J. Prosen, W. H. Johnson, and Florence Y. Pergiel, J. Research NBS 62, 43 (1959) RP2927. 


\section{Appendix}

\subsection{Apparatus}

The major items of apparatus used in bombcalorimetric measurements on fuels at the National Bureau of Standards are listed in table 2. On the basis of prices listed in manufacturers' catalogs at the present time (1959), the estimated total cost of replacement of the equipment listed is in the neighborhood of $\$ 15,000$.

TABLE 2. Major items cf equipment used in measurement of heats of combustion of liquid fuels at the National Bureau of Standards

\begin{tabular}{|c|c|}
\hline $\begin{array}{l}\text { Mueller bridge. } \\
\text { Resistance thermometer. } \\
\text { Galvanometer. } \\
\text { Calorimeter, jacket, and thermo- } \\
\text { stat. } \\
\text { Combustion bornb. } \\
\text { Platinum crucibles and fittings. } \\
\text { Balance. } \\
\text { Set of class M weights for above. } \\
\text { Heary duty balance }\end{array}$ & $\begin{array}{l}\text { Set of weights for above. } \\
\text { Oxygen purifier. } \\
\text { Temperature controller for oxygen } \\
\text { purifier. } \\
\text { Laboratory table for bridge. } \\
\text { Laboratory bench. } \\
\text { Pellet press. } \\
\text { Reducing valve. } \\
\text { Julius suspension. }\end{array}$ \\
\hline
\end{tabular}

The approximate arrangement of the calorimeter and accessory apparatus is shown in figure 4 . The reading station $R$ provides support for the lamp $L$ and ground glass scale $S$, as well as for some electrical equipment for measurements not covered in this Monograph. It also contains a convenient "desk" for use in recording the data of bomb calorimetric experiments. The arrangement of the equipment for purifying oxygen and admitting it to the bomb is shown schematically in figure 5 .

The arrangements shown in figures 4 and 5 are not necessarily the best under all circumstances. For example, the bench $B E$ (fig. 4) is considerably larger than necessary for the calorimeter alone, as part of this table is used for other purposes.

The essential features of the various items of apparatus are described below. Items not obtainable commercially, and modifications of commercial items are described in considerable detail.

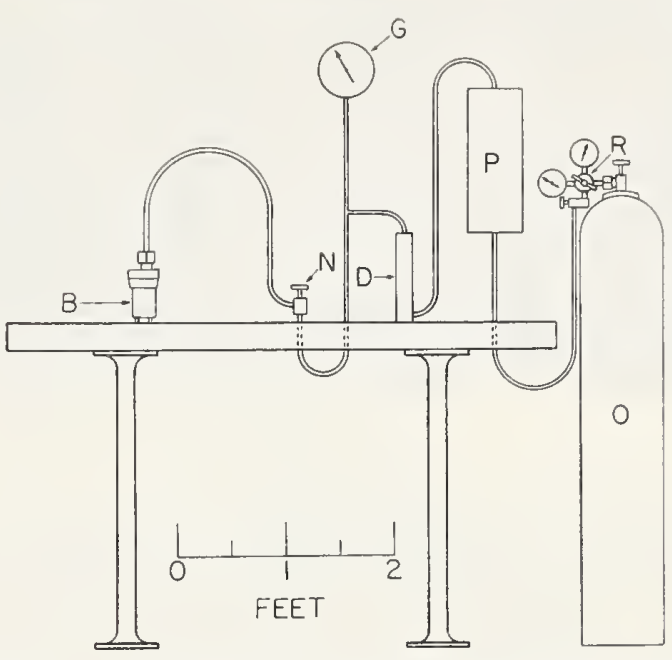

F1GURE 5. Arrangement of apparatus for admitting oxygen to bomb.

$\mathrm{B}$, bomb; $\mathrm{D}$, dryer; $\mathrm{G}$, pressure gage; $\mathrm{N}$, ncedle valve; $\mathrm{O}$, oxygen cylinder; $P$, oxygen purifier; and $R$, reducing valve.

\section{a. Bridge}

The bridge used is a special Mueller bridge designed especially for bomb-calorimetric measurements. It is substantially equivalent to the commercially available bridge designated as $G-2$. In addition to the usual 10-, 20-, 30-ohm coils of the $10 \times 10$-ohm decade this bridge has a 20.5 -ohm coil. Such a coil is useful in bomb-calorimetric measurements, where the change in resistance of the thermometer in an experiment is of the order of $0.3 \mathrm{ohm}$, since it makes it possible to avoid a change in setting of the dial of the $10 \times 1-\mathrm{obm}$ decade which would otherwise be necessary in certain circumstances. Thus if the initial and final resistances of the thermometer are 27.8721
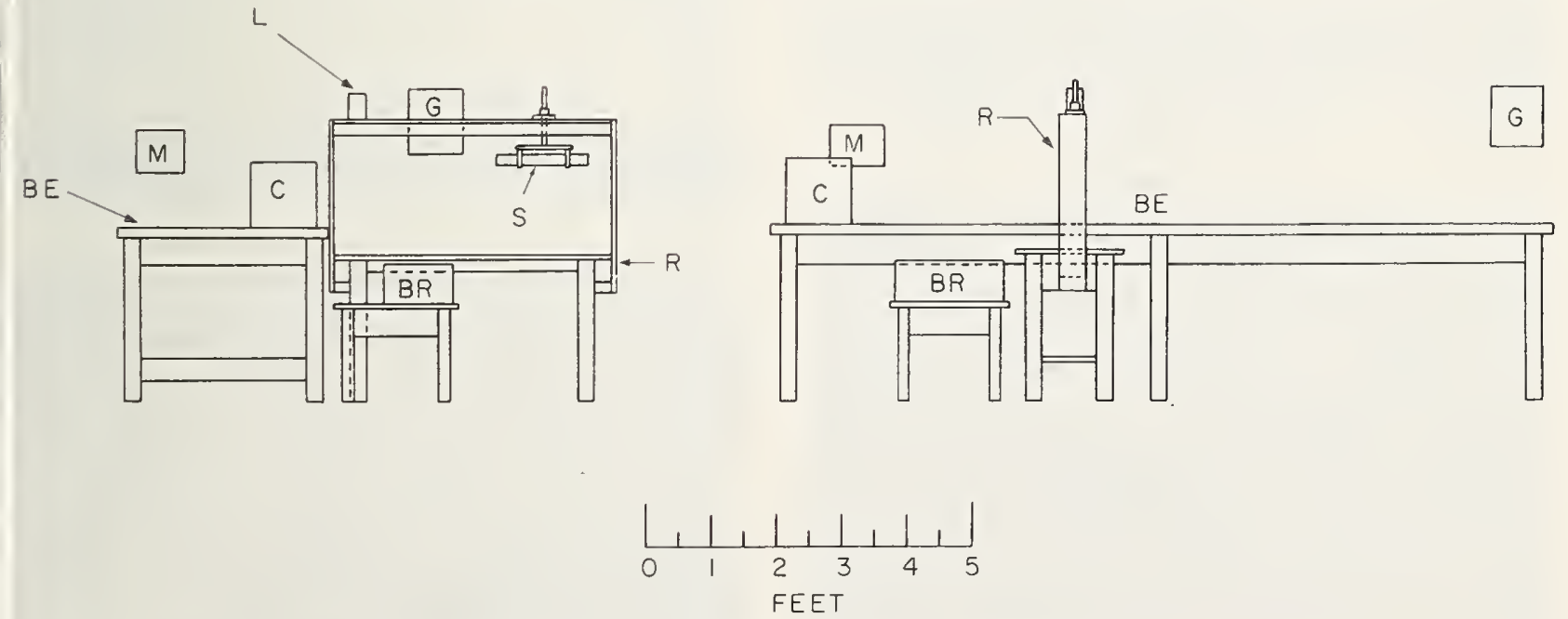

FIGURE 4. Arrangement of calorimetric apparatus.

$\mathrm{BE}$, bench; C, calorimeter; $\mathrm{BR}$, bridge; $\mathrm{G}$, galvanometer; L, light source; $\mathrm{M}$, stirring motor; $\mathrm{R}$, reading station; and $\mathrm{S}$, ground glass scale. 
and 28.1806, the corresponding dial settings with the 20.5-ohm coil would be $20.5+7.3721$ and $20.5+7.6806$ and the change in dial setting would involve only the $10 \times 0.1$ ohm and lower decades. If no $20.5-\mathrm{ohm}$ coil were available, it would be necessary to change the dial setting of the $10 \times 1$ ohm decade from 7 to 8 at a time when the temperature of the calorimeter was rising very rapidly. This would greatly increase the difficulty of following the temperature of the calorimeter during the period of rapid temperature rise, and might also involve a larger correction to the observed change in resistance of the thermometer.

\section{b. Thermometer}

The platinum resistance thermometer has a resistance of approximately $25.5 \mathrm{ohms}$ at $0^{\circ} \mathrm{C}$. It is of the strain-free type with coil windings very close to the wall of the enclosing glass tube. It was purchased under a specified requirement that the temperature of the coil with a current of $1 \mathrm{ma}$ flowing through it should not exceed that of the bath in which the thermometer is immersed by more than $0.0003^{\circ} \mathrm{C}$. Calorimetric resistance thermometers enclosed in metal sheaths are available commercially. These are less subject to breakage than glass-enclosed thermometers, but are subject to damage from bending the metal sheath. Such damage might not be obvious on casual examination, but could be detected by periodic determination of the ice point of the thermometer. 福数:

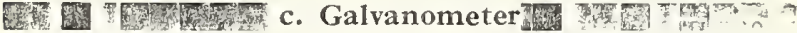

The galvanometer has a sensitivity of about $0.2 \mu \mathrm{v} / \mathrm{mm}$ at $1 \mathrm{~m}$, an external critical damping resistance of 40 ohms and a period of about 5 sec. The damping resistance used is so chosen that the galvanometer is slightly underdamped. The galvanometer is protected from air currents and abrupt changes in temperature by an enclosure made of cork pipe covering (for 6 in. pipe) about $10 \mathrm{in}$. in outside diameter. The galvanometer is supported by a Julıus suspension, which is very effective in reducing the effect of vibrations.

\section{d. Bomb}

The combustion bomb ${ }^{21}$ is made of a corrosionresistant alloy (Illium). It is provided with a check valve for admitting oxygen and also with a needle valve for releasing the gases contained in it. It is sealed by a self-sealing neoprene gasket. The original bomb has been modified by replacing the three supporting feet furnished with it by new ones of monel metal about $12 \mathrm{~mm}$ in height. This permits freer circulation of water under the bomb.

The base-metal electrode inside of the bomb, and the crucible-support rod were cut off leaving lengths of about $3 \mathrm{~cm}$ from the point at which they enter the lock nuts. To the shortened electrode

21 Parr Instrument Company Catalog No. 1101. was hard soldered a 1/16 in. platinum rod about 3 $\mathrm{cm}$ in length. To the shortened crucible-support rod was hard soldered a 5 -cm length of $1 / 8$ in. o.d. platinum tube having a 0.01 in. wall. The arrangement of electrode, crucible support, crucible, electric fuse and $0.3 \mathrm{~mm}$ platinum leads thereto is shown in figure 6(a) (valves not shown). An enlarged view of the platinum crucible is shown at (c) figure 6. The crucible is put in place by sliding the split tube $(5 / 32$ in. o.d.) shown at the right of figure 6 (c) over the end of the $1 / 8 \mathrm{in}$. platinum crucible-support tube referred to above until the fuse is nearly in contact with the sample of combustible. The split tube may be deformed slightly with the fingers to make it fit the $1 / 8$ in. tube tightly enough to be held in place by friction. The length of the crucible-support, and the position of the crucible on this support should be such that the crucible cannot come in contact with the water in the bottom of the bomb when the bomb is assembled. If the crucible should come in contact with the water, combustion will be incomplete.

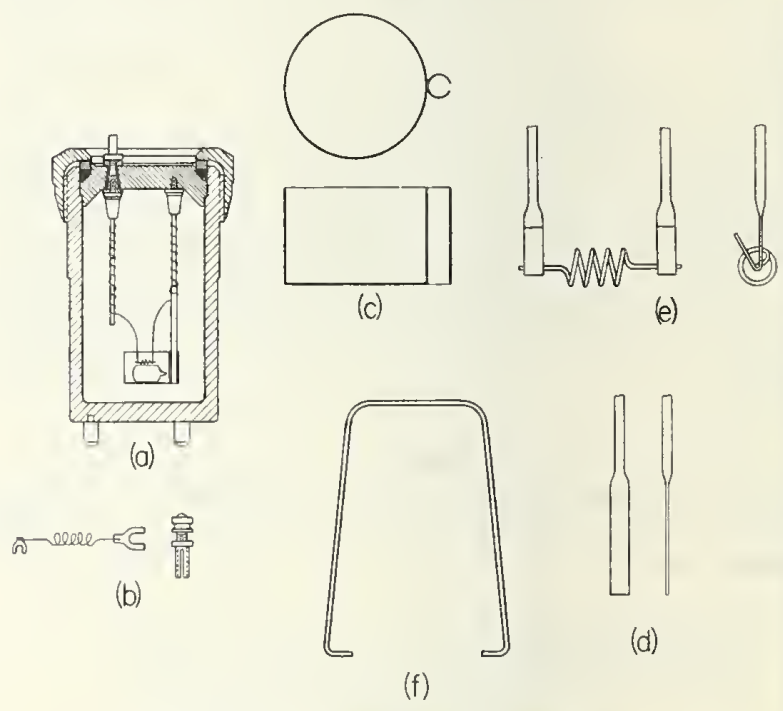

Figure 6. Bomb and accessories.

(a) Bomb, showing arrangement of crucible, crucible support, electrode, etc. (Valves not shown, see fig. 1); (b) Binding post and lead wire; (c) Enlarged view of crucible; (d) Platinum lead flattened at end; (e) Fuse wire attached to platinum leads; and (f) Hook for handling bomb.

A supply of platinum lead wire is wound around the electrode and crucible support rods as shown, and a sufficient amount of this to reach down to the crucible is unwound for each experiment. The electric fuse is a 2-cin length of Parr fuse wire (No. $34 \mathrm{AWG}$ chromel C) which is formed into a small helix by winding it around a rod about 0.04 in. in diameter. The method of attaching the fuse to the platinum leads is illustrated at (d) and (e), figure 6. The ends of the lead wires are first flattened by hammering as shown at (d). The flattened ends are then bent and the fuse inserted as shown at (e), after which the turned-up ends 
of the lead wires are hammered down with a small hammer or squeezed together with pliers to clamp the ends of the fuse wires.

The terminal nut attached to the electrode outside of the bomb was replaced by one of the form shown at (a), figure 6 . The binding post shown at (b), figure 6 , has a split tube at its lower end which can be slipped over the upper part of the terminal nut, where it fits tightly so as to make good electrical contact. The lead wire shown at (b), figure 6, connects this binding post to one on the calorimeter jacket which is connected in the firing circuit. This wire is soldered to spade-type terminals at each end, the larger of which normally is permanently attached to the binding post shown at (b). This wire should be so insulated as to aroid any electrical contact with the calorimeter vessel. With alternating current the contact of the lead wire and bomb electrode with the water in the calorimeter does not result in any appreciable electrical leakage, provided actual metallic contact with the calorimeter ressel is aroided.

\section{e. Calorimeter and Jacket}

The calorimeter now used for fuel testing is of the type described by Dickinson [8]. The calorimeter vessel is of the form shown at (a) and (b), figure 1. The calorimeter cover is in the form of a flat circular disk with vertical wall about $1 \mathrm{~cm}$ in height at the edge. The holes OT and OL (fig. 1 (b)) are surrounded by metal collars about $1 \mathrm{~cm}$ high. The corer fits tightly enough inside of the calorimeter ressel so that it is held in place by friction. Its lower surface is in contact with the water of the calorimeter. The calorimeter ressel is supported inside of the jacket br pins of stainless steel, in the manner described by Dickinson $[8]$.

The Dickinson type calorimeter was constructed in the NBS shops. There is a commercially arailable "submarine" calorimeter, ${ }^{22}$ based on a design developed at the National Bureau of Standards [20], which has been found to be substantially equivalent in performance to the Dickinson calorimeter. The submarine calorimeter is supplied without a thermostat for the jacket bath, so that a thermostat for controlling the bath temperature to a few thousand ths of a degree must be purchased or constructed.

The thermostat ${ }^{23}$ used with the particular Dickinson trpe calorimeter used for fuel testing is similar to one previously described by Osborne, Stimson, and Sligh [9]. "It consists of a "bulb" filled with toluene and connected to a glass capillary U-tube (1.5 mm i.d.) containing mercury. Expansion of the toluene as a l'esult of rising tem-

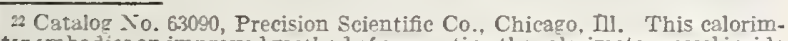
eter embodies an improved method of supporting the calorimeter Tessel inside of the jacket [20].

23 Temperature-control units for which a control accuracy of $0.001^{\circ} \mathrm{C}$. is claimed are arailable commercially. Numerous other temperature-control devices are described in the literature. See for example the papers cited in reference [3]. For an temperature-control unit it is important that the relative locations of heater, stirrer, and temperature-sensing element should be as shown in figure 1, that is, the bath liquid should flow from the heater, through the stirring propeller, and orer the temperature-sensitire element by the shortest path possible. perature causes the mercury to make contact with a needle closing an electrical circuit through an electronic relay ${ }^{24}$ which shuts off the current in the electric heater in the jacket bath. The "bulb" $T B$ (fig. 1) consists of about $3 \mathrm{~m}$ of $3 / 16$ in. copper tubing with 0.01 in. Wall thickness wound into a helix and so located in the same tubular housing as the jacket bath stirrer that the water flows orer the bulb immediately after leaving the stirrer. The glass U-tube and attached valve for admitting or removing toluene are shown in figure 7 . The ralve has tubular openings at the top and bottom for attaching the reservoir $B$ and the capillary U-tube as described below.

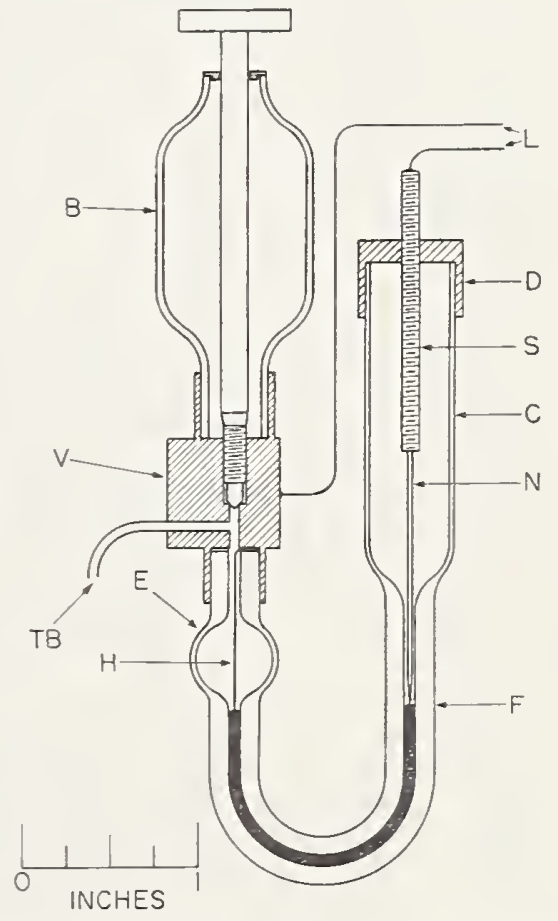

FIGURE T. Valve and glass parts of thermostat.

$\mathrm{V}$, valre; $\mathrm{TB}$, tube to thermostat bulb; $\mathrm{B}$, toluene reserroir; $\mathrm{F}$, capillary $U$-tube; $E$, enlargement in capillary; $E$, platiuum wire; $L$, leads to relay; C, Prrex tube; D, brass cap; S, screw; and $\mathrm{X}$, steel needle.

The Pyrex reservoir $B$ contains a supply of toluene which is separated from that in the "bulb" $T B$ (fig. 1) by the valve $V$ when it is closed. This valve is opened when the calorimeter is shut down thus permitting toluene to flow from the reservoir into the bulb as the bath cools. Failure to open the valve when the bath cools usually results in air being dramn into the system through the capillary $F$ (fig. 7). The Pyrex bulb $C$ (fig. 7) also contains toluene which protects the mercury surface in the capillary from oxidation. The enlargement $E$ in the capillary is larger in volume than the mercury in the capillary so that no mercury will be drawn into the metal parts of the system if the valve $V$ is inadvertently left closed when the calorimeter is shut down.

\footnotetext{
24 American Instrument Company Catalog No. 4-5301.
} 
Electrical connection between the Valve $V$ and the mercury in the capillary $U$-tube is provided by a platinum wire $H$, long enough to reach from the top of the capillary to near the bottom of the $U$. The upper end of the wire is flattened and bent at a right angle to the rest of the wire so as to insure electrical contact with the valve.

The glass reservoir $B$ and the capillary glass $U$ tube are soldered into the tubular openings in the brass valve $V$ with pure tin following the procedure described by Meyers [10].

To fill the thermostat with toluene the tube $C$ (with cap $D$ removed) is connected to a large bulb $G$ above $C$ (at least $250 \mathrm{~cm}^{3}$, not shown in fig. 7) by means of a short piece of Tygon tubing. Somewhat more than enough toluene to fill the thermostat is placed in the bulb $G$, and some is also placed in the reservoir $B$. Air can be removed from the thermostat system by heating the "bulb" (TB, fig. 1) to about $100^{\circ} \mathrm{C}$ and repeatedly evacuating the bulb $G$ above the toluene and then admitting air. To test for air in the system, air is admitted above the toluene in $G$, the valve $V$ is opened, the toluene meniscus is brought into the capillary at $F$, and the valve is closed. If the system is free of air, moderate air pressure applied by mouth above the toluene will not cause a movement of the meniscus in the capillary of more than 0.2 or $0.3 \mathrm{~mm}$. The removal of air from the system should be done before introducing mercury into the capillary. If air is inadvertently admitted to the system after mereury has been introduced into the capillary, the mercury should be removed before attempting to remove the air.

In preparing for a calorimetric experiment the jacket is first heated to the desired temperature, the heating current is reduced to slightly more than enough to maintain the temperature constant, and the valve $T$ is closed. Final adjustment of the temperature can be made by moving the needle $N$ up or down by rotating the cap $D$ relative to the screw $S$ (fig. 7). The heating current should be adjusted so that the on and off periods are about equal in length.

The method of bringing the firing leads through the jacket is illustrated in figure $1(\mathrm{c}) .{ }^{25}$ Two metal rods, one of which is shown at $R$, are cemented with wax into tubes $T$ which pass through the jacket, so that the rods are insulated from the jacket. Each rod is drilled and tapped at each end for a 4-40 brass screw, which is used to clamp the terminal of the lead wire between the two washers, $W$, thus forming a binding post at each end of the rod. The terminal at the left end of the lead wire shown in figure $1(\mathrm{~d})$ is attached permanently to one of the inner binding posts and the clip at the other end of the wire (d) is shipped over the edge of the calorimeter ressel when it is

${ }_{23}$ This method is not applicable to some types of calorimeters, for example, submarine calorimeters. The important feature of the method is that the leads arc in good thermal contact with the jacket, but are electrically insulated from it. assembled preparatory to making an experiment. The other lead wire, from the binding post on the bomb (see fig. 6(b)), is attached to the other inner binding post after the bomb has been put in the calorimeter and the calorimeter cover has been put in place. The two outer binding posts are connected through an ammeter and a switch to a small transformer having a secondary voltage of 8 or 10 . The switch should be of the momentary contact push-button type normally open except when held closed by the operator.

\section{f. Balance for Weighing Samples}

The balance used for weighing samples of combustibles is an undamped semimicro balance (capacity $100 \mathrm{~g}$ ) having a sensitivity reciprocal of $0.14 \mathrm{mg}$ per division on the index scale. The balance is provided with a keyboard arrangement for adding and removing weights from 1 to $100 \mathrm{mg}$. Temperature gradients paralkel to the balance beam are reduced by covering the walls of the balance case inside and out with aluminum foil, and by locating the balance where temperature gradients in the room are small. It has been found, however, that best results are obtained with the balance when there is a vertical temperature gradient such that the top of the balanee case is warmer by 0.03 to $0.1^{\circ} \mathrm{C}$ than the bottom.

The weights used with the balance are highgrade one-piece (class M) weights and were calibrated in the Mass and Scale Section of the National Bureau of Standards.

For most purposes a good analytical balance would be satisfactory if provided with high grade calibrated weights, and properly used.

\section{g. Balance for Weighing Calorimeter}

This is a magnetically damped heavy duty balance having a capacity of $5 \mathrm{~kg}$ and a sensitivity of about $0.5 \mathrm{mg}$. The tantalum-plated weights used with this balance are of good quality but are not calibrated. Calibration was considered unnecessary since the purpose of weighing the calorimeter is not to determine its absolute weight, but to reproduce the same weight of calorimeter plus water for each experiment. For this purpose it is important that the weights remain constant in value, and that the same weights be used at all times, but the actual masses of the weights used need not be known accuratcly.

\section{h. Oxygen Purifier}

Combustible impurities in the oxygen are removed by passing it through a cylinder containing cupric oxide maintained at a temperature of $500^{\circ} \mathrm{C}\left(932^{\circ} \mathrm{F}\right)$. The purifier ${ }^{26}$ consists essentially of a heavy-walled alloy-steel cylinder having inside dimensions of approximately 1 in. diameter by $10 \mathrm{in}$. length, and capable of withstanding a pressure of at least 2500 psi at $500^{\circ} \mathrm{C}$. The cylinder is provided with an electric heater. It

\footnotetext{
26 American Instrument Company 1942 Catalog No, 406-31.
} 
has inlet and outlet at opposite ends, and a well for inserting a thermocouple for measurement of temperature. The cylinder is filled with wire-form cupric oxide. The temperature and oxygen pressure in the purifier should not be allowed to rise above values consistent with safety. The manufacturers recommendations regarding safety precautions should be adhered to rigidly. "The strength of materials decreases rapidly with increasing temperature.

The temperature of the purifier can be controlled by means of a "Fail safe" controller. Such controllers are available commercially. ${ }^{27}$ The pressure is easily controlled by a reducing valve attached to the oxygen cylinder.

\section{i. Laboratory Table for Bridge}

This table should be large enough to accommodate the bridge comfortably, and of a height which will afford convenience in the manipulation of the bridge dials. A table 22 in. square by $18 \mathrm{in}$. high is convenient for use with a $\mathrm{G}-2$ bridge as it allows space for batteries, etc

\section{j. Pressure Gage}

The Bourdon pressure gage used has a $6 \mathrm{in}$. dial and is graduated from 0 to 800 psi in steps of 10 psi, so that it can be read to about 1 or 2 psi. The gage was calibrated in the Mechanical Instruments Section of the NBS and was set so as to read correctly at 450 psi.

\subsection{Glass Sample Bulbs}

For measurements of heat of combustion of kerosenes, gasolines, or other volatile liquids it is necessary, in order to permit accurate weighing of the sample and to insure that it is all in the liquid state until it is ignited in the bomb, to enclose the sample in a thin-walled glass bulb, so constructed and filled that it will not break under the pressure of the oxygen. The type of bulb ${ }^{28}$ used in testing of volatile fuels at the National Bureau of Standards, and the method of making such bulbs are illustrated in figure 8 . Attempts to obtain bulbs of this type from manufacturers of glass laboratory apparatus have been unsuccessful to date, and such bulbs are therefore made in our own laboratory. A brief description of the method of making the bulbs will be given below. It should be recognized, however, that it is virtually impossible to describe the method in such detail as to enable an inexperienced worker to make bulbs successfully on his first attempt. The best that can be done is to outline the method, and to depend upon the individual worker to develop the necessary techniques by trial. It is to be expected that in the early attempts failures will far outweigh successes, and that a reasonable degree of skill can be acquired only by long and patient practice.

\footnotetext{
27 For example American Instrument Company Catalog No. 49-9570. Arthur S. Lapine and Company Catalog No. 357-70.

23 Bulhs of this type were first deseribed by Richards and Barry [11].
}

The starting material for making bulbs is soft glass tubing 4 to $6 \mathrm{~mm}$ in outside diameter. ${ }^{29}$ Approximately $2 \mathrm{~cm}$ of such a tube is heated in a moderately liot air-gas flame of an ordinary laboratory blast burnel. When the glass becomes soft it is removed from the flame and drawn down to a fine capillary having a minimum outside diameter of about $1 \mathrm{~mm}$. This procedure is then repeated in such a manner as to leave a short section of the same diameter as that of the original tube, as shown at figure 8(a). The two capillaries are then broken at about their midpoints, leaving a piece such as that shown at figure $8(b)$. The remaining glass-working operations are carried out with an air-gas micro blast burner capable of producing relatively small flames. Using such a micro burner the piece illustrated at (b), figure 8, is rotated and heated by a small hot flame at the point indicated by the arrows, and is then drawn down to $1 \mathrm{~mm}$ outside diameter. This procedure is repeated on the other side of the enlargement in the tube, the final product being as illustrated at (c), figure 8. This piece is then rotated and heated by a small sharp flame at the center of the enlargement as indicated by the arrow at (c), and the two halves of the piece are pulled apart as shown at (d), figure 8, where the enlarged end of the piece at the left has been reduced in size by removal of surplus glass. This removal of surplus glass is accomplished by heating the end of the enlargement until it softens, and then pulling off small filaments of glass until an enlargement of the proper size remains. After such removal of surplus glass from both of the pieces shown at (d) a spherical bulb, not greater than $14 \mathrm{~mm}$ in diameter, is blown on each piece (fig. 8(e)). The spherical bulb is then flattened on one side by holding the bulb above a small soft flame (no primary air) about $1 / 4 \mathrm{in}$. in diameter by $1 / 4 \mathrm{in}$. high, and gradually lowering it until the glass on the under side of the bulb softens and flattens. This procedure is then repeated so as to flatten the opposite side of the bulb. The final form of the bulb is sliown at figure $8(\mathrm{f})$. The operations of bringing the bulb down on to the flame and removing it after flattening is complete should be done gradually, since too rapid heating or cooling of the bulb may cause it to break.

The most difficult part of the procedure just described is that of blowing a bulb of the proper size. Success in this operation depends in part upon having the proper amount of glass on the end of the tube (fig. 8 (d)) and in part upon the type of flame used, as well as upon the procedure followed in blowing the bulb. A flame which has been found to be satisfactory is one about $1 / 4$ in. in diameter by $5 / 8$ in. in height with just enough primary air so that there is a small yellow tip in the flame. The end of the tube (fig. $8(\mathrm{~d})$ ) on which the bulb is to be blown is heated in the

\footnotetext{
${ }^{29}$ Pyrex glass has been found unsatisfactory for this purpose, as unburned carbon is usually found on such glass after a combustion. This may be related to the fact that the Pyrex glass does not melt completely wheu the sample is burned, as does soft glass, and there fore partially retains its original form, thus inhibiting access of oxygen to the fuel.
} 


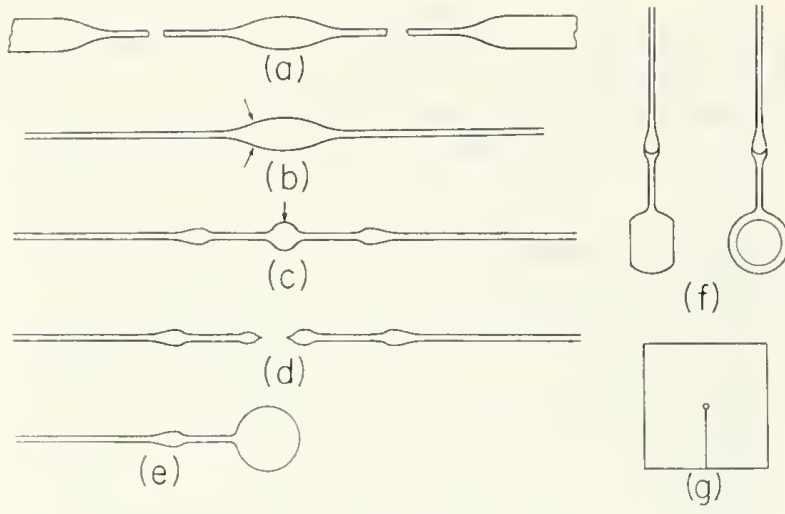

Figure 8. Steps in making sample bulbs.

flame until sufficiently hot, and is then removed from the flame and the bulb blown immediately. In order to prevent sagging of the end of the tube when heated, the tube is held at an angle of about $30^{\circ}$ to the vertical and is rotated about its axis continuously. Usually the first few attempts to blow a bulb will be unsuccessful because the initial size of the bulb is so small that a relatively high pressure will be required to overcome surface tension and the viscous resistance to flow of the glass. If the pressure is not high enough the bulb will expand so slowly at first that the glass will harden before the desired size is reached. If the pressure is high enough to expand the bulb at a sufficiently high initial rate, the bulb may suddenly expand to too large a size before the glass hardens. The ideal procedure would be to blow very hard at first and then less so as the bulb becomes larger. This is very difficult to do because the whole operation must be carried out in a very short time before the glass hardens. It is therefore usually more successful to repeat several times the procedure of blowing the bulb to a diameter of 4 or $5 \mathrm{~mm}$ and then shrinking it by heating, before attempting to blow the bulb to the required size. This procedure also serves to provide a somewhat more uniform distribution of glass in the final bulb.

Too large an amount of glass in the bulb and too hot a flame are both conducive to too large size of the bulb. Too small an amount of glass and too low a flame temperature have the opposite effect. Success in blowing a suitable bulb of the proper size depends upon the proper combination of flame temperature, amount of glass and technique of blowing.

The spherical bulb should be not more than 14 $\mathrm{mm}$ in diameter, and the completed bulb should not contain more than about $1.3 \mathrm{~cm}^{3}$ nor less than $0.7 \mathrm{~cm}^{3}$ of liquid. The flat sides of the bulb should be thin enough to deflect visibly, but not so thin as to break, under moderate air pressure applied by mouth. The weight of the glass in the bulb after sealing off the stem near the bulb should be between 0.05 and $0.10 \mathrm{~g}$. The outside diameter of the capillary adjacent to the bulb should be between 1.0 and $1.3 \mathrm{~mm}$. The inside diameter should be at least $0.5 \mathrm{~mm}$ and preferably a little larger in order to permit easy insertion of a No. 27 hypodermic needle $(0.4 \mathrm{~mm}$ diam.) in filling the bulb. The appearance of the flat sides of a completed bulb will give some indication as to whether it will be satisfactory. Usually the flat sides present a scalloped appearance, although if the amount of glass in the bulb is greater than normal they may be nearly plane. If the scallops are very fine, say $1 \mathrm{~mm}$ or less apart, the bulb is probably too thin and fragile to be satisfactory.

Samples of liquid fuel can be conveniently introduced into the previously weighed glass bulbs by means of a hypodermic syringe fitted with a No. 27 needle. The liquid should be introduced into the bulb slowly in order to permit escape of the air initially present without undue increase in pressure. The bulb should be filled completely so that the liquid extends up into the enlargement in the stem as indicated at figure 8(f). Usually a small bubble will be trapped in the bulb at the point where it joins on to the stem, but such a bubble can usually be removed without too much difficulty with the aid of the hypodermic needle or a piece of wire. In filling the bulbs the usual safety precautions for the handling of volatile organic solvents should be taken, particularly if the fuel contains tetraethyl lead [13].

In filling bulbs with very volatile liquid fuels such as gasolines, great care should be taken to avoid loss of material by evaporation, as this causes fractionation of the material with a consequent change in heat of combustion. The container should be kept tightly closed at all times except when withdrawing a sample with the hypodermic syringe. The sample can be withdrawn more quickly if the needle is detached from the syringe. In replacing the needle after withdrawal of the sample care should be taken to avoid contact of gasoline with the hands, particularly if it contains tetraethyl lead.

After the bulb is filled, the capillary is sealed off so as to leave a stem about 3 or $4 \mathrm{~mm}$ long attached to the bulb. In order to do this the bulb must be cooled so as to draw the liquid out of the capillary, and the bulb must be protected from the flame during the sealing operation. To protect the bulb from the flame a small piece of asbestos paper with a hole in the center and with a cut between the hole and the edge (fig. $8(\mathrm{~g})$ ) is slipped over the capillary via the cut so that the capillary goes through the hole, and the piece of asbestos paper is in contact with the bulb. The bulb is then cooled by placing it on a mass of shaved ice in a beaker, or on a copper plate in contact with ice, and when the meniscus in the capillary disappears below the asbestos paper, the capillary is sealed off with a small hot air-gas flame and removed from the bulb. The bulb should then be removed promptly from the ice to avoid possible breakage due to 
excessire contraction of the liquid. The filled bulb is conveniently handled by the short projecting capillary by means of forceps of the type ordinarily used for handling balance weights. Particles of asbestos which may adhere to the bulb should be removed by means of a camel's hair brush. Any liquid remaining in the capillary detached from the bulb in sealing it should be removed before weighing the filled bulb together with this detached capillary. If the liquid is sufficiently volatile it can be removed by flaming the capillary several times with the micro-burner flame. The flame should be applied first to the closed end of the capillary, and moved gradually to the open end in such a manner as to avoid softening the glass. If the liquid is of low volatility, it should be washed out with a volatile solvent, such as benzene or petroleum ether, introduced into the capillary by means of a hypodermic syringe before flaming as described above.

When first sealed, the bulb may contain a small bubble of air. This will dissolve in the liquid after a time leaving the bulb completely filled with liquid. Bulbs prepared in the manner described should withstand changes in room temperature of $10^{\circ} \mathrm{C}$ or more, and pressures of at least $30 \mathrm{~atm}$ (450 psi) without breaking.

Glass sample bulbs of different types have been described by a number of workers [16, 17].

Washington, April 14, 1959. 



\section{THE NATIONAL BUREAU OF STANDARDS}

The scope of activities of the National Bureau of Standards at its major laboratories, in Washington, D.C., and Boulder, Colo., is suggested in the following listing of the divisions and sections engaged in technical work. In general, each section carries out specialized research, development, and engineering in the field indicated by its title. A brief description of the activities, and of the resultant publications, appears on the inside front cover.

WASHINGTON, D.C.

Electricity and Electronics. Resistance and Reactance. Electron Devices. Electrical Instruments. Magnetic Measurements. Dielectrics. Engineering Electronics. Electronic Instrumentation. Electrochemistry.

Optics and Metrology. Photometry and Colorimetry. Optical Instruments. Photographic Technology. Length. Engineering Metrology.

Heat. Temperature Physics. Thermodynamics. Cryogenic Physics. Rheology. Molecular Kinetics. Free Radicals Research.

Atomic and Radiation Physics. Spectroscopy. Radiometry. Mass Spectrometry. Solid State Physics. Electron Physics. Atomic Physics. Neutron Physics. Radiation Theory. Radioactivity. X-ray. High Energy Radiation. Nucleonic Instrumentation. Radiological Equipment.

Chemistry. Organic Coatings. Surface Chemistry. Organic Chemistry. Analytical Chemistry. Inorganic Chemistry. Electrodeposition. Molecular Structure and Properties of Gases. Physical Chemistry. Thermochemistry. Spectrochemistry. Pure Substances.

Mechanics. Sound. Mechanical Instruments. Fluid Mechanics. Engineering Mechanics. Mass and Scale. Capacity, Density, and Fluid Meters. Combustion Controls.

Organic and Fibrous Materials. Rubber. Textiles. Paper. Leather. Testing and Specifcations. Polymer Structure. Plastics. Dental Research.

Metallurgy. Thermal Metallurgy. Chemical Metallurgy. Mechanical Metallurgy. Corrosion. Metal Physics.

Mineral Products. Engineering Ceramics. Glass. Refractories. Enameled Metals. Constitution and Microstructure.

Building Technology. Structural Engineering. Fire Protection. Air Conditioning. Heating and Refrigeration. Floor, Roof, and Wall Coverings. Codes and Safety Standards. Heat Transfer. Concreting Materials.

Applied Mathematics. Numerical Analysis. Computation. Statistical Engineering. Mathematical Physics.

Data Processing Systems. SEAC Engineering Group. Components and Techniques. Digital Circuitry. Digital Systems. Analog Systems. Applications Engineering.

- Office of Basic Instrumentation.

- Office of Weights and Measures.

\section{BOULDER, COLORADO}

Cryogenic Engineering. Cryogenic Equipment. Cryogenic Processes. Properties of Materials. Gas Liquefaction.

Radio Propagation Physics. Upper Atmosphere Research. Ionosphere Research. Regular Prediction Services. Sun-Earth Relationships. VHF Research. Radio Warning Services. Airglow and Aurora. Radio Astronomy and Arctic Propagation.

Radio Propagation Engineering. Data Reduction Instrumentation. Radio Noise. Tropospheric Measurements. Trophospheric Analysis. Propagation-Terrain Effects. Radio-Meteorology. Lower Atmosphere Physics.

Radio Standards. High-Frequency Electrical Standards. Radio Broadcast Service. Radio and Microwave Materials. Electronic Calibration Center. Microwave Physics. Microwave Circuit Standards.

Radio Communication and Systems. Low Frequency and Very Low Frequency Research. High Frequency and Very High Frequency Research. Modulation Systems. Antenna Research. Navigation Systems. Systems Analysis Field Operations. 
